\title{
BALANCED FERTILIZATION OF NITROGEN AND MICRONUTRIENTS FOR WHEAT GROWN IN SALT AFFECTED SOILS
}

\author{
R. A. El-Dissoky (1), R. A. Ramadan ${ }^{(2)}$ and I. A. A. Hegab(1) \\ Soils, Water and Environment Res. Inst., Agric. Res. Center. Giza, Egypt. \\ Wheat Res. Dept., Field Crops Res. Inst., Agric. Res. Center. Giza, Egypt. \\ R.eldissoky@yahoo.com
}

Received: Feb. 13, 2017

Accepted: Mar. 6, 2017

\begin{abstract}
The balanced fertilization become necessary for sustainable agriculture under conditions of old and newly soils. Moreover the balanced fertilization under stress conditions especially salinity stress becomes more important. Micronutrients are part of many crucial physiological plant processes which plants suffered from their uptake in salted affected soils, and combined application of $N$ and micronutrients helps in improving plant growth, yield and its quality. So, two field experiments were conducted at salt affected soils at Tag Al-Ezz Research station (Latitude 30.9 68.6' N, Longitude 31.6 96.5" E), Dakahlia Governorate, Egypt, during growing winter seasons of 2014/2015 and 2015/2016, to evaluate the effect of applying rates of $N$ fertilizer (50, 75 and $100 \%$ of recommended $N$ "i.e. $75 \mathrm{~kg} \mathrm{~N} \mathrm{fed}^{11 ")}$ along with foliar application of micronutrients in chelated form; Fe- EDTA, Zn- EDTA and Mn-EDTA ( $F_{0}$ : control, $F_{1}$ : two sprays at tillering and elongation stages and $F_{2}$ : three sprays at tillering, elongation and heading stages) on wheat yield and its components, grain quality, NPK and micronutrients contents as well as K/Na ratio in straw and grain. Results showed that all parameters of wheat yield were significantly increased with foliar application of micronutrients at the three stages (tillering, elongation and heading stages), under nitrogen fertilization rate $100 \%$ of recommended $N\left(75 \mathrm{~kg} \mathrm{Ned}^{-1}\right)$. Also, the results reveal that grain quality, i.e. 1000-grain weight, protein \%, P, Fe and $\mathrm{Zn}$ concentrations significantly increased with nitrogen rates and foliar micronutrients applications. The uptake of macro and micro nutrients increased with increasing $N$ rate and number of sprays micronutrients. Plant growth improved under the condition of salt affected soil with foliar spraying micronutrients and the highest $N$ rate $(75 \mathrm{~kg} \mathrm{~N}$ $\mathrm{fed}^{1}$ ), and this was clear from plant height, straw and grain yields and concentrations of $K$ and $\mathrm{Na}$ and $\mathrm{K} / \mathrm{Na}$ ratio. Interaction between the highest $\mathrm{N}$ rate and foliar spray of micronutrients at three growth stages recorded the highest mean of grain yield (19.24 ardab fed $\left.{ }^{1}\right)$ and straw yields $\left(5368 \mathrm{~kg} \mathrm{fed}^{-1}\right)$.
\end{abstract}

Key words: Micronutrients, nitrogen, salt affect soils and wheat yield.

\section{INTRODUCTION}

Wheat (Triticum aestivum, L.) is the first strategic cereal crop in Egypt, and there is a gap between production and consumption. Increasing wheat production could be achieved through maximizing the yield per unit area or invading deserts to expand the cultivated area and/or raise the productivity of salinity soils that is one of the major problems that face Egyptian agriculture, because of semiarid climate and shortage of irrigation water, which return on productivity of crops that decreased in these soils. Salinity can change the micronutrient concentrations in plants, depending upon the type of crop species and levels of salinity (Sharpley, et al., 1992). Hu and Schmidhalter (2001) found that the effects of salinity on concentrations of $\mathrm{Mn}, \mathrm{Zn}, \mathrm{Fe}$ and $B$ in plants are complex, and the changes in $\mathrm{Mn}, \mathrm{Zn}, \mathrm{Fe}$ and $\mathrm{B}$ concentrations under saline conditions depended upon the levels of macronutrients and salinity and the plant organs. Faizy, et al., (2010) showed that 
grain yield of wheat significantly decreased by about $13.74 \%$ and $24.11 \%$ and straw yield by about $23.46 \%$ and $32.85 \%$ with increasing soil salinity levels to 5.4 and 10 $\mathrm{dSm}^{-1}$, respectively. Ghogdi et al., (2012) found that salinity stress $\left(5,10,15 \mathrm{dSm}^{-1}\right)$ decreased $\mathrm{K}^{+}$content, $\mathrm{K}^{+} / \mathrm{Na}^{+}$ratio and grain yield; however $\mathrm{Na}^{+}$content in all the genotypes of wheat were increased. They also, showed that the salinity tolerance in tolerant cultivars as clearly by lower decrease in grain yield is associated with the lower sodium accumulation and higher $\mathrm{K}^{+} / \mathrm{Na}^{+}$compared to the sensitive cultivars.

Fertilization plays a vital role under salinity conditions for improving plant productivity, especially foliar fertilization. As mentioned by many researchers, the efficiency of foliar fertilization is higher than that of soil fertilizer application in these situations. El-Fouly et al., (2001) noted that application of micronutrients to wheat showed positive effects on growth and nutrients uptake under treatment of salinity. They concluded that foliar application of micronutrients could enhance salinity tolerance. Ling and Silberbush, (2002) illustrated that foliar nutrients application under drought and salinity conditions may be able to exclude or include water. Sairam and Tyagi (2004) suggested that foliar spraying with micronutrients, especially $\mathrm{Fe}$, $\mathrm{Mn}$ and $\mathrm{Zn}$ increased yield crops and mineral contents of many plants types under saline stress conditions.

Many researches provided the importance of micronutrients; Mengle and Kirkby (2001) showed that micronutrients are essential elements for plant life particularly under limited condition. Iron ( $\mathrm{Fe})$, zinc $(\mathrm{Zn})$, copper $(\mathrm{Cu})$ and manganese $(\mathrm{Mn})$ are essential micronutrients for plants and humans, and a deficiency of just one of these nutrients can greatly reduce plant yield and even cause plant death. Micronutrient deficiency, especially $\mathrm{Fe}$ and $\mathrm{Zn}$ deficiency is widespread in humans (FAO, 2002).
Abd El-Magid (2001) found that the mixture of $\mathrm{Zn}+\mathrm{Fe}+\mathrm{Mn}$ gave highest protein content in leaves and kernels as well as yield of wheat and barley. In field study in salt affected soils, Nassar et al., (2004) found that to obtain the highest economic wheat grain and straw yields, wheat plants must fertilized with the optimum rate of $\mathrm{N}$ $\left(75 \mathrm{~kg} \mathrm{~N} \mathrm{fed}^{-1}\right)$, they also showed that foliar spray of micronutrients promote the use efficiency of $\mathrm{N}$-fertilization, and reflected on grain and straw yields and gave better nutritive content than the control (plants fertilized with recommended dose of $\mathrm{N}$ and did not spray with micronutrients). Abu ElFotoh et al., (2006) reported that application of $75 \mathrm{~kg} \mathrm{~N} \mathrm{fed}^{-1}+100 \mathrm{~kg}$ single super phosphate $+50 \mathrm{~kg}$ potassium sulphate fertilizer in combination with foliar application of $0.2 \mathrm{~g} / \mathrm{L}$ of each chelate $\mathrm{Zn}, \mathrm{Fe}$ and $\mathrm{Mn}$ caused significant increment in wheat grain yield under salinity clayey soil condition. Also, they showed that wheat grain yield increased by $19 \%$ with foliar micronutrients.

Marschner (1995) reported that $\mathrm{Zn}$ deficient plants reduced the rate of protein synthesis and protein content drastically but increased the accumulation of amino acids. Negm and Zahran (2001) found that foliar application of $1 \mathrm{~g}$ mixture $1: 1: 1 / \mathrm{L}$ of $\mathrm{Fe}$ chelate, Zn-chelate and Mn-chelate at booting stage only or along with either tillering or elongation stage had the most significant effect on increasing wheat grain and straw yields on sandy soils. In field experiment on clay loam soil, Habib (2009) showed that foliar application of $\mathrm{Zn}$ and $\mathrm{Fe}$ at tillering and heading stages increased grain yield and its quality compared with control, since $\mathrm{Zn}$ concentration increased from 12.17 to $20.27 \mathrm{mg} \mathrm{kg}^{-1}$ and $\mathrm{Fe}$ concentration increased from 84.93 to 139.6 mg. $\mathrm{kg}^{-1}$. Also, Zeidan et al., (2010) indicated that grain yield, straw yield, 1000grain weight and number of grains/spike, $\mathrm{Fe}$, $\mathrm{Mn}$ and $\mathrm{Zn}$ concentrations in grain and protein content were significantly increased by foliar application of micronutrients (1\% $\mathrm{FeSO}_{4}, 0.5 \% \mathrm{ZnSO}_{4}$ and $0.5 \% \mathrm{MnSO}_{4}$ ) at 
tillering and heading stages. Moreover, Mahrous et al., (2010) in field study under saline soil condition, showed that twice foliar spray with mixture of $0.3 \mathrm{~g} \mathrm{Zn}$-EDTA+ $0.3 \mathrm{~g}$ $\mathrm{Mn}-\mathrm{EDTA}+0.3 \mathrm{~g} \mathrm{Fe}-\mathrm{EDTA}+100 \mathrm{~kg} \mathrm{~N} / \mathrm{fed}$ as ammonium nitrate+ $100 \mathrm{~kg}$ calcium super phosphate $+50 \mathrm{~kg}$ potassium sulfate +10 $\mathrm{m}^{3}$ compost/fed recorded the highest values of wheat growth, yield and mineral concentration of $\mathrm{N}, \mathrm{P}, \mathrm{K}, \mathrm{Fe}, \mathrm{Zn}$ and $\mathrm{Mn}$ in grains.

Abd El-Hadi (2015) showed that foliar application of $\mathrm{Zn}, \mathrm{Mn}$ and Fe-chelates increased wheat grain yield by $0.65 \mathrm{t} / \mathrm{fed}$ and straw yield by $1.2 \mathrm{t} / \mathrm{fed}$, however the increases in grain and straw yields were lower by spraying $\mathrm{Fe}$ and $\mathrm{Mn}$ chelates. Also, he showed that grain yield increases ranged from 3-12 \%. Foliar application of plant nutrients especially micro-nutrients proved to be useful for several plants species in soils of high $\mathrm{pH}$ values as that in Egyptian soils. Wheat is one of the crops which show a great response to foliar application (Abd El-Hadi, 2015). Bakry et al., (2016) showed that foliar application of $4.5 \mathrm{~g} / \mathrm{L}$ zinc-chelate gave the best results for wheat grain yield, protein $\%$ and harvest index. Also, Ezatollah et al., (2016) showed that foliar spray of zinc sulphate $(2 \mathrm{~g} / \mathrm{L})$ at different wheat growth stages increased grain yield, number of grains/spike, plant height and grain contents of zinc and ascorbic acid.

Increasing $\mathrm{Zn}$ and $\mathrm{Fe}$ concentration of food crop plants, resulting in better crop production and improved human health is an important global challenge. Zinc deficiency is currently listed as a major risk factor for human health and causes of death globally. Cereals are the major source of $\mathrm{Zn}$ for the world's population chiefly for the poor people of the third world countries (WHO, 2002 and FAO, 2002). Stepien and Wajtkowiak (2016) illustrated that mineral NPK fertilization, combined with micronutrients $(\mathrm{Cu}+\mathrm{Zn}+\mathrm{Mn})$ increased $\mathrm{Cu}$ and $\mathrm{Zn}$ content in grain by $22.6 \%$ and $17.7 \%$ respectively.
So, the main objectives of this investigation were to achieve to the balanced fertilization of nitrogen and micronutrients which improve growth and productivity of wheat grown in salt affected soils.

\section{MATERIALS AND METHODS}

Two field experiments were conducted at Tag Al-Ezz Agricultural Research StationAgricultural Research Center (Latitude $30.9^{\circ}$ 68.6' N, Longitude 31.6 96.5" E)- Dakahlia Governorate, Egypt, during winter growing seasons of $2014 / 2015$ and 2015/2016 to evaluate the effect of different $\mathrm{N}$ rates and foliar spray of micronutrients in chelated form (Fe-EDTA, Zn-EDTA and Mn-EDTA) at different growth stages for achievement to balanced fertilization which recognize the optimum yield the highest quality and nutrients uptake under salt affected soil.

Data in Table (1) show some soil properties of the experimental field before sowing, according to Jackson (1967) and Page (1982).

Wheat variety Sakha 93 was sown on 20 November 2014, and harvested on 5 May 2015 in $1^{\text {st }}$ season, while it was sown on 15 November 2015 and harvested on $1^{\text {st }}$ May 2016 in $2^{\text {nd }}$ season. The two experiments were designed in split plot design with three replicates; plot area was $10.5 \mathrm{~m}^{2}$. The main plots were assigned to three $\mathrm{N}$ rates i.e. 50 , 75 and $100 \%$ of recommended $\mathrm{N}$ rate (I,e, $\mathrm{N}_{1}: 37.5, \mathrm{~N}_{2}: 56.25$ and $\mathrm{N}_{3}: 75 \mathrm{~kg} \mathrm{~N} \mathrm{fed}^{-1}$ ). The sub plots were for treatments of foliar micronutrients spraying; $F_{0}$ : control (without foliar spraying), $F_{1}$ : two foliar sprays at tillering (after 30 days from sowing) and elongation (after 45-50 days) stages and $F_{2}$ : three foliar sprays at tillering, elongation and heading (after 70-75 days) stages. Spraying solution volume was $200 \mathrm{~L}$ fed $^{-1}$. Micronutrient concentrations were $0.3 \mathrm{~g} \mathrm{~L}^{-1}$ from each nutrient chelate (Fe-EDTA: $13 \%$ Fe, Zn-EDTA: $14 \% \mathrm{Zn}$ and Mn-EDTA: $13 \%$ $\mathrm{Mn}$ ) with concentration of $39 \mathrm{mg} \mathrm{Fe} \mathrm{L}^{-1}, 42$ $\mathrm{mg} \mathrm{Zn} \mathrm{L}^{-1}$ and $39 \mathrm{mg} \mathrm{Mn} \mathrm{L}^{-1}$. 
El-Dissoky, et al.,

Table 1: Some chemical and physical properties of the soil before sowing.

\begin{tabular}{|c|c|c|c|c|c|c|c|}
\hline \multicolumn{2}{|c|}{ Properties } & $\begin{array}{c}1^{\text {st }} \\
\text { Season }\end{array}$ & $\begin{array}{c}2^{\text {nd }} \\
\text { Season }\end{array}$ & \multirow{2}{*}{\multicolumn{2}{|c|}{$\begin{array}{l}\text { Properties } \\
{ }^{*} \mathrm{EC}\left(\mathrm{dSm^{-1 }}\right)\end{array}$}} & $\begin{array}{c}1^{\text {st }} \\
\text { Season }\end{array}$ & $\begin{array}{c}2^{\text {nd }} \\
\text { Season }\end{array}$ \\
\hline \multirow{4}{*}{$\begin{array}{l}\text { particles } \\
\text { size } \\
\text { distribution }\end{array}$} & Sand \% & 17.75 & 18.60 & & & 6.06 & 5.15 \\
\hline & Silt \% & 36.80 & 35.15 & \multirow{4}{*}{$\begin{array}{c}\text { Soluble } \\
\text { Cations } \\
\left(\text { meq L }^{-1}\right)\end{array}$} & $\mathrm{Ca}^{++}$ & 14.19 & 12.85 \\
\hline & Clay \% & 45.45 & 46.25 & & $\mathrm{Mg}^{++}$ & 10.40 & 8.60 \\
\hline & Texture & Clay & Clay & & $\mathrm{Na}^{+}$ & 33.45 & 27.70 \\
\hline \multicolumn{2}{|c|}{ SP $\%$} & 60.0 & 63.0 & & $\mathrm{~K}^{+}$ & 2.56 & 2.35 \\
\hline \multicolumn{2}{|c|}{ O.M \% } & 0.43 & 0.57 & \multirow{4}{*}{ 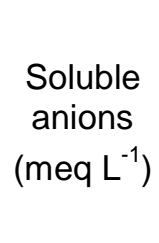 } & $\mathrm{CO}_{3}^{--}$ & -- & -- \\
\hline \multicolumn{2}{|c|}{$\mathrm{CaCO}_{3} \%$} & 1.35 & 1.77 & & $\mathrm{HCO}_{3}^{-}$ & 1.98 & 1.90 \\
\hline \multicolumn{2}{|c|}{ * $\mathrm{pH}$} & 8.28 & 8.28 & & $\mathrm{Cl}^{-}$ & 37.00 & 30.10 \\
\hline \multicolumn{2}{|c|}{ ESP } & $6.05 \%$ & $5.80 \%$ & & $\mathrm{SO}_{4}^{--}$ & 21.62 & 19.50 \\
\hline \multicolumn{4}{|c|}{ Available $\mathrm{N}, \mathrm{P}$ and $\mathrm{K}\left(\mathrm{mg} \mathrm{kg}^{-1}\right)$} & \multicolumn{4}{|c|}{ Available $\mathrm{Fe}, \mathrm{Zn}$ and $\mathrm{Mn}\left(\mathrm{mg} \mathrm{kg}^{-1}\right)$} \\
\hline \multicolumn{2}{|c|}{$\mathrm{N}$} & 23 & 25 & \multicolumn{2}{|c|}{$\mathrm{Fe}$} & 2.0 & 2.0 \\
\hline \multicolumn{2}{|c|}{$\mathrm{P}$} & 9 & 10 & \multicolumn{2}{|c|}{$\mathrm{Zn}$} & 0.75 & 0.80 \\
\hline \multicolumn{2}{|c|}{ K } & 270 & 280 & \multicolumn{2}{|c|}{$\mathrm{Mn}$} & 1.6 & 1.7 \\
\hline
\end{tabular}

${ }^{*} \mathrm{pH}$ : determined in 1:2.5 soil : water suspension, $\quad{ }^{* *} \mathrm{EC}$ : determined in soil paste extract.

Phosphorus was applied to all plots as calcium super phosphate fertilizer $(15 \%$ $\left.\mathrm{P}_{2} \mathrm{O}_{5}\right)$ at the rate of $200 \mathrm{~kg} \mathrm{fed}^{-1}\left(30 \mathrm{~kg} \mathrm{P}_{2} \mathrm{O}_{5}\right.$ $\mathrm{fed}^{-1}$ ) before sowing. Potassium was also applied to all plots as potassium sulphate $\left(48 \% \mathrm{~K}_{2} \mathrm{O}\right)$ at the rate of $50 \mathrm{~kg} \mathrm{fed}^{-1}(24 \mathrm{~kg}$ $\mathrm{K}_{2} \mathrm{O}$ fed $\left.^{-1}\right)$ at sowing. $\mathrm{N}$-fertilizer rates $\left(\mathrm{N}_{1}\right.$ : $37.5, \mathrm{~N}_{2}: 56.25$ and $\mathrm{N}_{3}: 75 \mathrm{~kg} \mathrm{~N}$ fed $^{-1}$ ) were applied as ammonium nitrate fertilizer (33.5 $\% \mathrm{~N}$ ), at two equal doses with first and second irrigation after sowing.

At harvest, plant height, number of spikes $/ \mathrm{m}^{2}$, spike length, biological yield, 1000 -grain weight, grain and straw yield parameters were recorded. Samples of grain and straw were taken for chemical analysis to determine nitrogen, phosphorus, potassium, sodium, iron, zinc and manganese concentrations according to Jackson, (1967), and then the uptake of these nutrients was calculated. The ratio between $\mathrm{K}$ and $\mathrm{Na}$ in straw and grain $(\mathrm{K} / \mathrm{Na}$ ratio) was calculated by dividing $\mathrm{K} \%$ over
$\mathrm{Na} \%$. Protein was calculated by multiplying " $\mathrm{N} \%$ in grains $\times 5.7 "$. Iron, zinc and manganese were determined using atomic adsorption plasma - ULTIMA 2- ICP- OES (Inductively Coupled Plasma Optical Emission Spectrometry) in the Unite of Soil and Water Analysis at Soils, Water and Environment Research Institute, ARC, Egypt.

The statistical analysis was estimated according to Gomez and Gomez (1984), and combined analysis was done over both seasons, then means values were compared against least significant differences test L.S.D. at $5 \%$.

\section{RESULTS AND DISCUSSION \\ Plant height and some yield components:}

Data in Table 2 show that plant height, spikes No. $/ \mathrm{m}^{2}$, spike length and 1000 -grain weight were significantly increased with increasing nitrogen rate (NR) up to $\mathrm{N}_{3}(100$ 
$\% \mathrm{~N})$. The recommended NR $\left(75 \mathrm{~kg} \mathrm{~N}^{\mathrm{fed}}{ }^{-1}\right)$ recorded the highest mean values of plant height $(93.3 \mathrm{~cm})$, spike No. $/ \mathrm{m}^{2}$ (289), spike length $(13.79 \mathrm{~cm})$ and 1000 grain weight $(41.8 \mathrm{~g})$. These results illustrate that the superiority was for the $100 \% \mathrm{~N}\left(\mathrm{~N}_{3}=75 \mathrm{~kg}\right.$ $\mathrm{N}$ fed $^{-1}$ ), and this result agreea with that obtained by Nassar et al., (2004), Mosaad et al., (2013) and El-Dissoky (2013).

As for the effect of interaction, it is obvious from the results in Table 2 that an integration effect was happened through the interaction between $\mathrm{N}$-rates (NR) and foliar spray of micronutrients. The interaction had a significant effect on 1000-grain weight, plant height, spike length and spike No. $/ \mathrm{m}^{2}$ in one season only, while the averages of the seasons for these parameters except spike length were significantly affected by this interaction. It is clear from the results that interaction between $\mathrm{N}_{3}$ and $\mathrm{F}_{2}$ had the highest mean values of plant height (95.6 $\mathrm{cm})$, spikes No. $/ \mathrm{m}^{2}$ (292), spike length $(14.33 \mathrm{~cm})$ and 1000 -grain weight $(42.3 \mathrm{~g})$. Also, these results are in accordance with those obtained by Nassar et al., (2004) and Mahrous et al., (2010) who found that foliar spray with mixture of $0.3 \mathrm{~g}$ of each of $\mathrm{Zn}$ EDTA, Mn-EDTA and Fe-EDTA with $\mathrm{N}$ fertilization recorded the highest values of wheat growth.

Table 2: Effect of nitrogen and micronutrients fertilization on plant height and some yield components of wheat grown in salted affected soils.

\begin{tabular}{|c|c|c|c|c|c|c|c|c|c|c|c|c|c|}
\hline \multirow{2}{*}{\multicolumn{2}{|c|}{$\begin{array}{l}\text { Characteristics } \\
\text { Treatments }\end{array}$}} & \multicolumn{3}{|c|}{$\begin{array}{l}\text { Plant Height } \\
(\mathrm{cm})\end{array}$} & \multicolumn{3}{|c|}{ No. of Spikes $/ \mathrm{m}^{2}$} & \multicolumn{3}{|c|}{$\begin{array}{l}\text { Spike Length } \\
(\mathrm{cm})\end{array}$} & \multicolumn{3}{|c|}{$\begin{array}{l}1000-\text { Grain weight } \\
\text { (g) }\end{array}$} \\
\hline & & $\begin{array}{c}1^{\text {st }} \\
\text { season }\end{array}$ & \begin{tabular}{|c|}
$2^{\text {nd }}$ \\
season
\end{tabular} & $\begin{array}{c}\text { Mean } \\
\text { (combine) }\end{array}$ & \begin{tabular}{|c|}
$1^{\text {st }}$ \\
season
\end{tabular} & $\begin{array}{l}2^{\text {nd }} \\
\text { season }\end{array}$ & Mean & \begin{tabular}{|c|}
$1^{\text {st }}$ \\
season
\end{tabular} & $\begin{array}{c}2^{\text {nd }} \\
\text { season }\end{array}$ & $\begin{array}{c}\text { Mean } \\
\text { (combine) }\end{array}$ & $\begin{array}{l}1^{\text {st }} \\
\text { season }\end{array}$ & \begin{tabular}{|l}
$2^{\text {nd }}$ \\
season
\end{tabular} & $\begin{array}{c}\text { Mean } \\
\text { (combine) }\end{array}$ \\
\hline \multicolumn{14}{|c|}{ Nitrogen rates effect: } \\
\hline \multicolumn{2}{|c|}{$\mathrm{N}_{1}$} & $74.8^{c}$ & $86.6^{c}$ & $80.7^{\mathrm{C}}$ & $266^{b}$ & $276^{\mathrm{C}}$ & $271^{\mathrm{C}}$ & $11.98^{\mathrm{C}}$ & $12.08^{c}$ & $12.03^{c}$ & $38.5^{c}$ & $38.7^{c}$ & $38.6^{c}$ \\
\hline \multicolumn{2}{|c|}{$\mathrm{N}_{2}$} & $84.4^{\mathrm{b}}$ & $93.8^{\mathrm{b}}$ & $89.1^{\mathrm{b}}$ & $280^{\mathrm{a}}$ & $282^{b}$ & $281^{\mathrm{b}}$ & $12.69^{b}$ & $13.19^{b}$ & $12.94^{\mathrm{b}}$ & $40.6^{b}$ & $41.2^{\mathrm{b}}$ & $40.9^{b}$ \\
\hline \multicolumn{2}{|c|}{$\mathrm{N}_{3}$} & $87.8^{\mathrm{a}}$ & $98.7^{\mathrm{a}}$ & $93.3^{\mathrm{a}}$ & $287^{\mathrm{a}}$ & $292^{\mathrm{a}}$ & $289^{\mathrm{a}}$ & $13.64^{a}$ & & $13.79^{\mathrm{a}}$ & $41.5^{\mathrm{a}}$ & $42.2^{a}$ & $41.8^{\mathrm{a}}$ \\
\hline \multicolumn{14}{|c|}{ Micronutrients effect: } \\
\hline \multicolumn{2}{|c|}{$F_{0}$} & $78.8^{\mathrm{c}}$ & $89.1^{\mathrm{c}}$ & $83.9^{c}$ & $270^{b}$ & $277^{b}$ & $274^{\mathrm{C}}$ & $12.22^{\mathrm{c}}$ & 12.3 & $12.30^{\mathrm{C}}$ & $39.3^{c}$ & $40.0^{c}$ & $39.6^{c}$ \\
\hline \multicolumn{2}{|c|}{$F_{1}$} & $83.1^{\mathrm{b}}$ & $93.8^{\mathrm{b}}$ & $88.4^{b}$ & $279^{a}$ & $285^{\mathrm{a}}$ & $282^{b}$ & $12.87^{b}$ & $13.19^{b}$ & $13.03^{b}$ & $40.3^{b}$ & $40.8^{b}$ & $40.5^{\mathrm{b}}$ \\
\hline \multicolumn{2}{|c|}{$\mathrm{F}_{2}$} & $85.2^{\mathrm{a}}$ & $96.1^{\mathrm{a}}$ & $90.6^{\mathrm{a}}$ & $283^{a}$ & $288^{a}$ & $285^{\mathrm{a}}$ & $13.22^{\mathrm{a}}$ & $13.64^{\mathrm{a}}$ & $13.43^{\mathrm{a}}$ & $41.0^{\mathrm{a}}$ & $41.2^{\mathrm{a}}$ & $41.1^{\mathrm{a}}$ \\
\hline \multicolumn{14}{|c|}{ Interaction effect: } \\
\hline \multirow{3}{*}{$\mathrm{N}_{1}$} & $\mathrm{~F}_{0}$ & $70.8^{f}$ & 83.0 & $76.9^{f}$ & $253^{\mathrm{e}}$ & 267 & $260^{\mathrm{e}}$ & 11.50 & $11.17^{\mathrm{e}}$ & 11.33 & $36.8 \mathrm{~g}$ & 37.9 & $37.3^{9}$ \\
\hline & $F_{1}$ & $73.7^{\mathrm{e}}$ & 86.8 & $80.3^{e}$ & $269^{d}$ & 280 & $275^{c d}$ & 12.03 & $12.37^{d}$ & 12.20 & $38.8^{f}$ & 38.7 & $38.7^{f}$ \\
\hline & $\mathrm{F}_{2}$ & $80.0^{d}$ & 89.8 & $84.9^{d}$ & $277^{\mathrm{cd}}$ & 282 & $279^{\text {cd }}$ & 12.40 & $12.70^{d}$ & 12.55 & $39.8^{\mathrm{e}}$ & 39.4 & $39.6^{\mathrm{e}}$ \\
\hline \multirow{3}{*}{$\mathrm{N}_{2}$} & $\mathrm{~F}_{0}$ & $80.5^{\mathrm{d}}$ & 89.5 & $85.0^{d}$ & $274^{d}$ & 277 & $276^{d}$ & 12.17 & $12.50^{d}$ & 12.33 & $40.1^{\mathrm{de}}$ & 40.5 & $40.3^{d}$ \\
\hline & $F_{1}$ & $86.8^{\mathrm{bc}}$ & 94.8 & $90.8^{\mathrm{bc}}$ & $281^{b c}$ & 283 & $282^{b c}$ & 12.77 & $13.17^{c}$ & 12.97 & $40.6^{\mathrm{cd}}$ & 41.4 & $41.0^{\mathrm{C}}$ \\
\hline & $\mathrm{F}_{2}$ & $85.8^{\mathrm{c}}$ & 97.0 & $91.4^{\mathrm{b}}$ & $284^{\mathrm{ab}}$ & 286 & $285^{\mathrm{b}}$ & 13.13 & $13.90^{\mathrm{b}}$ & 13.52 & $41.1^{\mathrm{bc}}$ & 41.6 & $41.4^{\mathrm{c}}$ \\
\hline \multirow{3}{*}{$\mathrm{N}_{3}$} & $\mathrm{~F}_{0}$ & $85.0^{\mathrm{C}}$ & 94.8 & $89.9^{c}$ & $284^{\mathrm{ab}}$ & 287 & $285^{b}$ & 13.00 & $13.47^{c}$ & 13.23 & $41.0^{\mathrm{bc}}$ & 41.6 & $41.3^{\mathrm{c}}$ \\
\hline & $F_{1}$ & $88.7^{\mathrm{ab}}$ & 99.8 & $94.3^{\mathrm{a}}$ & $288^{\mathrm{ab}}$ & 293 & $291^{a}$ & 13.80 & $14.03^{a b}$ & 13.92 & $41.5^{\mathrm{ab}}$ & 42.3 & $41.9^{\mathrm{b}}$ \\
\hline & $\mathrm{F}_{2}$ & $89.8^{\mathrm{a}}$ & 101.3 & $95.6^{\mathrm{a}}$ & $289^{a}$ & 295 & $292^{\mathrm{a}}$ & 14.13 & $14.33^{\mathrm{a}}$ & 14.23 & $41.9^{a}$ & 42.7 & $42.3^{\mathrm{a}}$ \\
\hline \multicolumn{14}{|c|}{ LSD at $5 \%:$} \\
\hline \multicolumn{2}{|c|}{ Nitrogen } & 1.14 & 1.98 & 0.54 & 7.24 & 1.82 & 3.16 & 0.24 & 0.18 & 0.17 & 0.5 & 0.5 & 0.42 \\
\hline \multicolumn{2}{|c|}{ Micronutrients } & 1.19 & 0.78 & 0.60 & 3.73 & 2.84 & 2.66 & 0.29 & 0.20 & 0.16 & 0.4 & 0.2 & 0.22 \\
\hline \multicolumn{2}{|c|}{ Interaction } & 2.05 & Ns & 1.05 & 6.46 & Ns & 4.61 & Ns & 0.36 & Ns & 0.8 & Ns & 0.38 \\
\hline
\end{tabular}




\section{Wheat Yield:}

Data obtained in Table 3 show that application of NR significantly increased wheat biological, grain and straw yields up to $\mathrm{N}_{3}$, since the differences between values of $N_{3}, N_{2}$ and $N_{1}$ were significant in both seasons. On average of the two seasons (combined analysis), grain yield increased by 26.81 and $42.40 \%$ with $\mathrm{N}$ fertilization rates $\mathrm{N}_{2}$ and $\mathrm{N}_{3}$ (75 and $100 \%$ of the recommended $\mathrm{N}$ ) compared with $\mathrm{N}_{1}(50 \%$ $\mathrm{N})$, also straw yield increased by 25.09 and $42.73 \%$, respectively. This result agreed with those obtained by Nassar et al., (2004), Moasad et al., (2013) and El-Dissoky et al., (2013).

Also, data reveal that foliar application of chelated micronutrients; Fe-EDTA, Zn-EDTA and Mn-EDTA had significant effect on wheat yield in both seasons. The highest means of biological yield $\left(7168 \mathrm{~kg} \mathrm{fed}^{-1}\right)$, grain yield (16.86 ard. fed $\left.{ }^{-1}\right)$ and straw yield $\left(4640 \mathrm{~kg} \mathrm{fed}^{-1}\right)$ were recorded with three foliar sprays of micronutrient treatment $F_{2}$ during the growth stages; tillering, elongation and heading, respectively. This result shows the importance of foliar micronutrient application at heading growth stage, and this may be attributed to improving the flowering and the translocation of carbohydrate and starch to grain which reflected on grain filling, 1000grain weight and grain yield. These results are in accordance with Zeidan et al., (2010), Abd El-Hadi (2015) and Ezatollah et al., (2016).

Table 3: Effect of nitrogen and micronutrients fertilization on yield of wheat grown in salted affected soils.

\begin{tabular}{|c|c|c|c|c|c|c|c|c|c|c|}
\hline \multirow{2}{*}{\multicolumn{2}{|c|}{$\begin{array}{l}\text { Characteristics } \\
\text { Treatments }\end{array}$}} & \multicolumn{3}{|c|}{$\begin{array}{l}\text { Biological yield } \\
\quad\left(\mathrm{kg} \mathrm{fed}^{-1}\right)\end{array}$} & \multicolumn{3}{|c|}{$\begin{array}{l}\text { Grain yield } \\
\left(\text { ard. fed }{ }^{-1}\right)\end{array}$} & \multicolumn{3}{|c|}{$\begin{array}{l}\text { Straw yield } \\
\left(\mathrm{kg} \mathrm{fed}^{-1}\right)\end{array}$} \\
\hline & & $\begin{array}{c}1^{\text {st }} \\
\text { season }\end{array}$ & $\begin{array}{c}2^{\text {nd }} \\
\text { season }\end{array}$ & $\begin{array}{c}\text { Mean } \\
\text { (combine) }\end{array}$ & $\begin{array}{c}1^{\text {st }} \\
\text { season }\end{array}$ & $\begin{array}{c}2^{\text {nd }} \\
\text { season }\end{array}$ & $\begin{array}{c}\text { Mean } \\
\text { (combine) }\end{array}$ & $\begin{array}{c}1^{\text {st }} \\
\text { season }\end{array}$ & $\begin{array}{c}2^{\text {nd }} \\
\text { season }\end{array}$ & $\begin{array}{c}\text { Mean } \\
\text { (combine) }\end{array}$ \\
\hline \multicolumn{11}{|c|}{ Nitrogen rates effect: } \\
\hline & & $5147^{C}$ & $5703^{c}$ & $5425^{c}$ & $12.27^{\mathrm{C}}$ & $13.38^{\mathrm{C}}$ & $12.83^{c}$ & $3305^{c}$ & $3697^{C}$ & $3501^{c}$ \\
\hline & & $6336^{\mathrm{b}}$ & $7303^{b}$ & $6819^{b}$ & $15.67^{b}$ & $16.86^{\mathrm{b}}$ & $16.27^{b}$ & $3984^{b}$ & $4773^{b}$ & $4379^{b}$ \\
\hline & & $7414^{\mathrm{a}}$ & $8060^{a}$ & $7737^{\mathrm{a}}$ & $17.68^{\mathrm{a}}$ & $18.86^{\mathrm{a}}$ & $18.27^{\mathrm{a}}$ & $4762^{\mathrm{a}}$ & $5232^{a}$ & $4997^{\mathrm{a}}$ \\
\hline \multicolumn{11}{|c|}{ Micronutrients effect: } \\
\hline & & $5738^{c}$ & $6396^{c}$ & $6067^{c}$ & $13.97^{\mathrm{C}}$ & $15.08^{C}$ & $14.53^{c}$ & $3642^{c}$ & $4134^{\mathrm{C}}$ & $3888^{c}$ \\
\hline & & $6329^{b}$ & $7163^{b}$ & $6746^{b}$ & $15.41^{b}$ & $16.55^{b}$ & $15.98^{b}$ & $4017^{b}$ & $4681^{b}$ & $4349^{b}$ \\
\hline & & $6830^{\mathrm{a}}$ & $7507^{\mathrm{a}}$ & $7168^{a}$ & $16.25^{\mathrm{a}}$ & $17.47^{\mathrm{a}}$ & $16.86^{\mathrm{a}}$ & $4393^{a}$ & $4887^{a}$ & $4640^{\mathrm{a}}$ \\
\hline \multicolumn{11}{|c|}{ Interaction effect: } \\
\hline \multirow{3}{*}{$\mathrm{N}_{1}$} & $\mathrm{~F}_{0}$ & $4493^{1}$ & $5144^{\mathrm{h}}$ & 4819 & 10.99 & $12.08^{\prime}$ & $11.53^{\prime}$ & $2845^{g}$ & $3332^{g}$ & 3089 \\
\hline & $\mathrm{F}_{1}$ & $5263^{h}$ & $5819^{g}$ & 5541 & 12.49 & $13.66^{\mathrm{h}}$ & $13.08^{h}$ & $3390^{f}$ & $3770^{\dagger}$ & 3580 \\
\hline & $\mathrm{F}_{2}$ & $5683^{g}$ & $6147^{f}$ & 5915 & 13.35 & $14.39^{9}$ & $13.87^{g}$ & $3681^{e}$ & $3988^{e}$ & 3835 \\
\hline \multirow{3}{*}{$\mathrm{N}_{2}$} & $\mathrm{~F}_{0}$ & $5883^{f}$ & $6561^{\mathrm{e}}$ & 6222 & 14.27 & $15.44^{\dagger}$ & $14.85^{\dagger}$ & $3743^{e}$ & $4246^{d}$ & 3994 \\
\hline & $\mathrm{F}_{1}$ & $6377^{e}$ & $7423^{d}$ & 6900 & 15.99 & $16.98^{e}$ & $16.49^{e}$ & $3978^{d}$ & $4876^{c}$ & 4427 \\
\hline & $F_{2}$ & $6747^{\mathrm{dc}}$ & $7923^{c}$ & 7335 & 16.76 & $18.17^{\mathrm{C}}$ & $17.46^{\mathrm{C}}$ & $4233^{c}$ & $5198^{b}$ & 4716 \\
\hline \multirow{3}{*}{$\mathrm{N}_{3}$} & $\mathrm{~F}_{0}$ & $6837^{c}$ & $7483^{d}$ & 7160 & 16.66 & $17.72^{d}$ & $17.19^{d}$ & $4338^{c}$ & $4825^{c}$ & 4581 \\
\hline & $\mathrm{F}_{1}$ & $7347^{b}$ & $8247^{b}$ & 7797 & 17.75 & $19.00^{b}$ & $18.37^{b}$ & $4685^{b}$ & $5397^{a}$ & 5041 \\
\hline & $\mathrm{F}_{2}$ & $8060^{\mathrm{a}}$ & $8450^{\mathrm{a}}$ & 8255 & 18.64 & $19.84^{\mathrm{a}}$ & $19.24^{\mathrm{a}}$ & $5263^{a}$ & $5473^{a}$ & 5368 \\
\hline \multicolumn{11}{|c|}{ LSD at $5 \%$ : } \\
\hline \multirow{3}{*}{\multicolumn{2}{|c|}{$\begin{array}{l}\text { Nitrogen } \\
\text { Micronutrients } \\
\text { Interaction }\end{array}$}} & 192.2 & 47.7 & 83.1 & 0.38 & 0.35 & 0.25 & 175.3 & 89.6 & 90.1 \\
\hline & & 105.8 & 79.2 & 65.3 & 0.22 & 0.17 & 0.12 & 103.3 & 74.7 & 61.5 \\
\hline & & 183.3 & 137.3 & Ns & Ns & 0.29 & 0.21 & 178.9 & 129.3 & Ns \\
\hline
\end{tabular}

ard. $=a r d a b=150 \mathrm{~kg}$. 
Concerning the effect of interaction, data in Table 3 and Fig. 1 show the significant effect of interaction on biological and straw yields in both seasons and grain yield in the $2^{\text {nd }}$ season. As for the combined analysis of the two seasons, it is obvious from the results that interaction between $\mathrm{N}$ rates and micronutrient significantly increased the yield of grain up to $19.24 \mathrm{ard}^{\text {a fed }}{ }^{-1}$ at $\mathrm{N}_{3} \times \mathrm{F}_{2}$ interaction. The highest values (combined analysis of two seasons) of biological yield $\left(8255 \mathrm{~kg} \mathrm{fed}^{-1}\right)$, grain yield (19.24 ard. $\left.\mathrm{fed}^{-1}\right)$ and straw yield (5368 $\mathrm{kg} \mathrm{fed}^{-1}$ ) were recorded under the interaction of $\mathrm{N}_{3}$ and $\mathrm{F}_{2}$. So, this result illustrate the integration of $\mathrm{N}$ fertilization and and foliar spray of micronutrients chelated Fe-EDTA, Zn-EDTA and Mn-EDTA at plant growth stages; tillering, elongation and heading. This may be attributed to the balance between nitrogen supply and micronutrient nutrition and plant requirements' in salt affected soils, and these results are agreed with El-Fouly et al., (2001), Hu and Schmidhalter (2001), Sairam and Tyagi (2004) and Nassar et al., (2004).

\section{Grain contents of protein, P, Fe and $\mathrm{Zn}$ (as grain quality):}

Data in Table 4 illustrate that wheat grain contents of protein, $\mathrm{P}, \mathrm{Fe}$ and $\mathrm{Zn}$ significantly improved with application of NR up to $N_{3}\left(75 \mathrm{~kg} \mathrm{~N}\right.$ fed $\left.^{-1}\right)$ and foliar spray of chelated micronutrients Fe-EDTA, Zn-EDTA and Mn-EDTA up to $F_{2}$ ( 3 sprays). It's obvious from the results that values protein $\%$, phosphorus \%, and iron and zinc concentrations were the highest with NR- $\mathrm{N}_{3}$ and $F_{2}$. Interaction between NR and treatments of micronutrients significantly affected grain $\mathrm{P}$ content while protein, $\mathrm{Fe}$ and $\mathrm{Zn}$ were insignificantly affected (as shown in Table 4 and Figs, 2 and 3 ). Application of NR- $\mathrm{N}_{3}\left(75 \mathrm{~kg} \mathrm{~N}\right.$ fed $\left.^{-1}\right)$ with 3 foliar sprays of Fe-EDTA, Zn-EDTA and MnEDTA had the highest mean values of protein $(16.18 \%), \mathrm{P}(0.428 \%), \mathrm{Fe}(128 \mathrm{mg}$ $\left.\mathrm{kg}^{-1}\right)$ and $\mathrm{Zn}\left(28.7 \mathrm{mg} \mathrm{kg}^{-1}\right)$. These results related with role of $\mathrm{N}$ in plant and the role of $\mathrm{Fe}, \mathrm{Zn}$ and $\mathrm{Mn}$ in plant that reflected on translocation of amino acids, protein and $\mathrm{Fe}$ and Zn. Nitrogen is Necessary for photosynthesis, formation of amino acids, protein, vitamins, Aids in production and use of carbohydrates; as well as micronutrients are a part of certain enzyme system, carbohydrate and starch formation and seed formation Mengle and Kirkby (2001). These results agreed with Negm and Zahran (2001), Habib (2009), Zeidan et al., (2010) and Ezatollah et al., (2016).

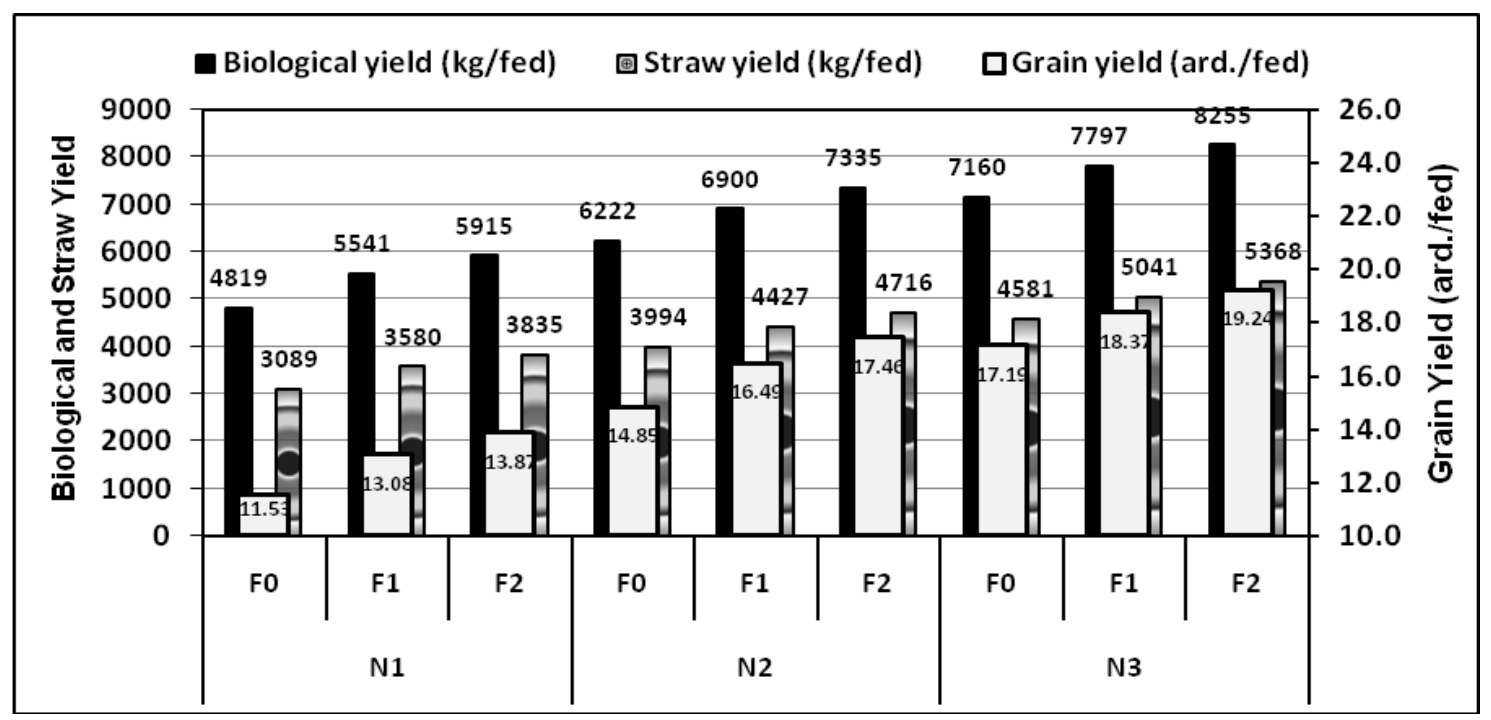

Fig. 1: Effect of interaction between NR and foliar micronutrients on wheat yield. 
El-Dissoky, et al.,

Table 4: Effect of nitrogen and micronutrients fertilization on grain contents of protein, $P$, Fe and $\mathrm{Zn}$ of wheat grown in salted affected soils.

\begin{tabular}{|c|c|c|c|c|c|c|c|c|c|c|c|c|c|}
\hline \multirow{2}{*}{\multicolumn{2}{|c|}{ Characteristics }} & \multicolumn{3}{|c|}{ Protein \% } & \multicolumn{3}{|c|}{$\mathrm{P} \%$} & \multicolumn{3}{|c|}{$\mathrm{Fe}\left(\mathrm{mg} \mathrm{kg}^{-1}\right)$} & \multicolumn{3}{|c|}{$\mathrm{Zn}\left(\mathrm{mg} \mathrm{kg}{ }^{-1}\right)$} \\
\hline & & season & season & $\begin{array}{c}\text { Mean } \\
\text { (combine) }\end{array}$ & season & season & $\begin{array}{l}\text { Mean } \\
\text { combine }\end{array}$ & $1^{\text {st }}$ & $\begin{array}{l}2^{\text {nd }} \\
\text { seasor }\end{array}$ & $\begin{array}{l}\text { Mean } \\
\text { combine }\end{array}$ & $\begin{array}{c}1^{\text {st }} \\
\text { season }\end{array}$ & season & $\begin{array}{l}\text { Mean } \\
\text { combine }\end{array}$ \\
\hline \multicolumn{14}{|c|}{ Nitrogen rates effect: } \\
\hline \multicolumn{2}{|c|}{$\mathrm{N}_{1}$} & \multicolumn{2}{|c|}{$14.04^{b} 14.89^{c}$} & $14.47^{\mathrm{C}}$ & $0.39^{b}$ & $0.40^{\mathrm{b}}$ & $0.39^{b}$ & $97^{b}$ & $92^{c}$ & $94^{\mathrm{C}}$ & $22.6^{c}$ & 21.5 & $22.0^{c}$ \\
\hline \multicolumn{2}{|c|}{$\mathrm{N}_{2}$} & $14.71^{b}$ & ${ }^{b} 15.26^{b}$ & $14.98^{b}$ & $0.42^{\mathrm{a}}$ & $0.42^{a}$ & $0.42^{\mathrm{a}}$ & $121^{a}$ & $103^{b}$ & $112^{b}$ & $26.6^{b}$ & 22.4 & $24.5^{\mathrm{b}}$ \\
\hline \multicolumn{2}{|c|}{$\mathrm{N}_{3}$} & $15.41^{\mathrm{a}}$ & ${ }^{\mathrm{a}} 15.94^{\mathrm{a}}$ & $15.67^{\mathrm{a}}$ & $0.42^{\mathrm{a}}$ & $0.43^{\mathrm{a}}$ & $0.42^{a}$ & $127^{\mathrm{a}}$ & $116^{a}$ & $121^{a}$ & $29.3^{a}$ & 23.4 & $26.3^{\mathrm{a}}$ \\
\hline \multicolumn{14}{|c|}{ Micronutrients effect: } \\
\hline \multicolumn{2}{|c|}{$\mathrm{F}_{0}$} & $13.72^{b}$ & ${ }^{b} 14.61^{b}$ & $14.17^{\mathrm{b}}$ & $0.40^{c}$ & $0.41^{\mathrm{b}}$ & $0.40^{c}$ & $107^{b}$ & $93^{c}$ & $100^{C}$ & $24.0^{c}$ & $17.8^{b}$ & $20.9^{b}$ \\
\hline \multicolumn{2}{|c|}{$F_{1}$} & $15.03^{a}$ & $\mathrm{a} 15.67^{\mathrm{a}}$ & $15.35^{\mathrm{a}}$ & $0.41^{\mathrm{b}}$ & $0.42^{a}$ & $0.42^{b}$ & $116^{a}$ & $105^{b}$ & $110^{b}$ & $26.8^{b}$ & $24.8^{a}$ & $25.8^{a}$ \\
\hline \multicolumn{2}{|c|}{$\mathrm{F}_{2}$} & $15.41^{a}$ & $\mathrm{a} 15.80^{\mathrm{a}}$ & $15.61^{a}$ & $0.41^{\mathrm{a}}$ & $0.42^{\mathrm{a}}$ & $0.42^{\mathrm{a}}$ & $121^{a}$ & $113^{a}$ & $117^{\mathrm{a}}$ & $27.7^{\mathrm{a}}$ & $24.7^{\mathrm{a}}$ & $26.2^{\mathrm{a}}$ \\
\hline \multicolumn{14}{|c|}{ Interaction effect: } \\
\hline \multirow{3}{*}{$\mathrm{N}_{1}$} & $\mathrm{~F}_{0}$ & 13.06 & 14.30 & 13.68 & $0.37^{e}$ & $0.38^{e}$ & $0.37^{f}$ & 89 & 82 & 85 & 21.0 & 16.7 & 18.8 \\
\hline & $F_{1}$ & 14.35 & 15.08 & 14.71 & $0.39^{d}$ & $0.40^{d}$ & $0.40^{\mathrm{e}}$ & 99 & 94 & 96 & 23.3 & 24.1 & 23.7 \\
\hline & $\mathrm{F}_{2}$ & 14.73 & 15.30 & 15.01 & $0.40^{c}$ & $0.41^{d}$ & $0.40^{d}$ & 103 & 101 & 102 & 23.5 & 23.7 & 23.6 \\
\hline \multirow{3}{*}{$\mathrm{N}_{2}$} & $\mathrm{~F}_{0}$ & 13.30 & 14.58 & 13.94 & $0.41^{b}$ & $0.42^{c}$ & $0.41^{c}$ & 110 & 94 & 102 & 24.9 & 17.6 & 21.3 \\
\hline & $F_{1}$ & 15.20 & 15.56 & 15.38 & $0.42^{\mathrm{ab}}$ & $0.43^{a b}$ & $0.42^{b}$ & 122 & 103 & 113 & 27.3 & 24.8 & 26.0 \\
\hline & $\mathrm{F}_{2}$ & 15.63 & 15.63 & 15.63 & $0.42^{\mathrm{a}}$ & $0.43^{a}$ & $0.42^{\mathrm{ab}}$ & 129 & 112 & 121 & 27.6 & 24.9 & 26.2 \\
\hline \multirow{3}{*}{$\mathrm{N}_{3}$} & $\mathrm{~F}_{0}$ & 14.80 & 14.96 & 14.88 & $0.41^{b}$ & $0.42^{b c}$ & $0.42^{c}$ & 122 & 104 & 113 & 26.0 & 19.2 & 22.6 \\
\hline & $F_{1}$ & 15.56 & 16.36 & 15.96 & $0.42^{a}$ & $0.43^{\mathrm{a}}$ & $0.43^{\mathrm{a}}$ & 127 & 117 & 122 & 29.8 & 25.5 & 27.7 \\
\hline & $\mathrm{F}_{2}$ & 15.87 & 16.49 & 16.18 & $0.42^{\mathrm{a}}$ & $0.43^{a}$ & $0.43^{\mathrm{a}}$ & 130 & 126 & 128 & 31.9 & 25.5 & 28.7 \\
\hline \multicolumn{14}{|c|}{ LSD at $5 \%:$} \\
\hline \multicolumn{2}{|c|}{ Nitrogen } & 0.42 & 0.26 & 0.15 & 0.009 & 0.007 & 0.005 & 6.25 & 8.38 & 4.84 & 0.87 & Ns & 1.41 \\
\hline \multirow{2}{*}{\multicolumn{2}{|c|}{$\begin{array}{l}\text { Micronutrients } \\
\text { Interaction }\end{array}$}} & 0.38 & 0.43 & 0.30 & 0.004 & 0.004 & 0.003 & 6.85 & 7.59 & 5.49 & 0.97 & 0.94 & 0.97 \\
\hline & & Ns & Ns & Ns & 0.007 & 0.007 & 0.005 & Ns & Ns & Ns & Ns & Ns & Ns \\
\hline
\end{tabular}

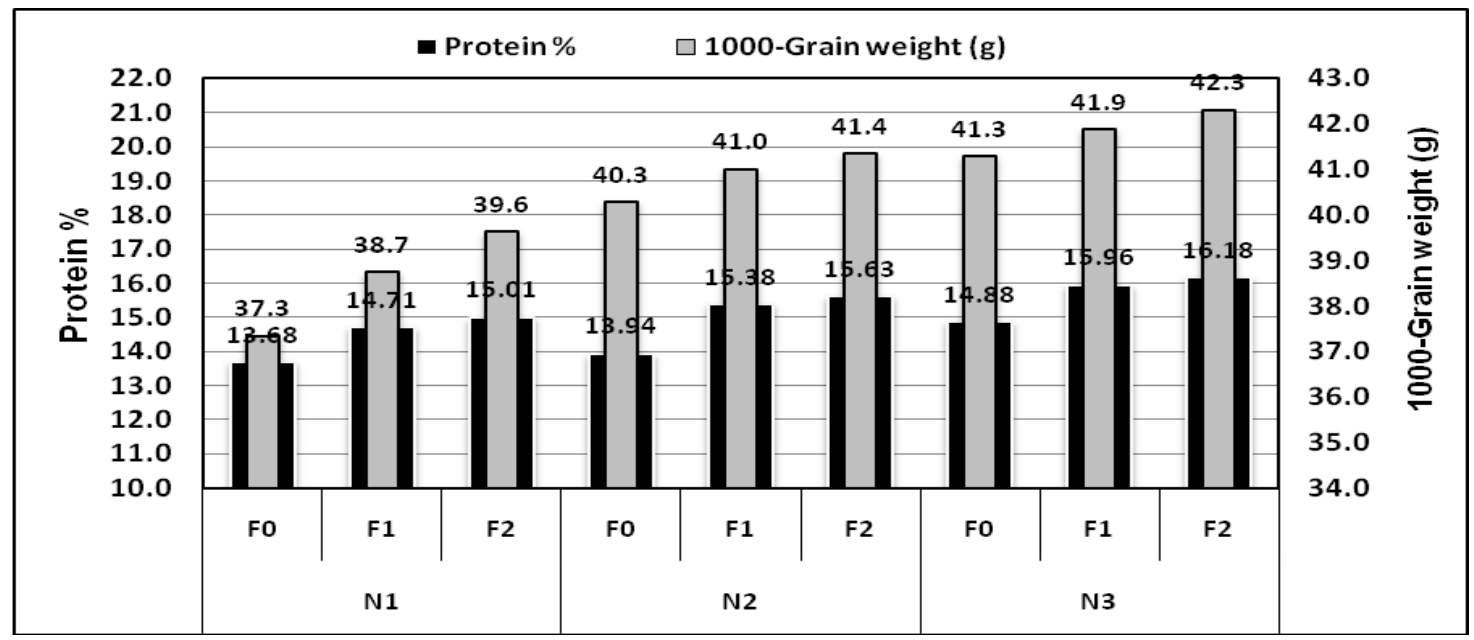

Fig. 2: Effect of interaction between NR and foliar micronutrients on protein $\%$ and 1000 grain weight. 


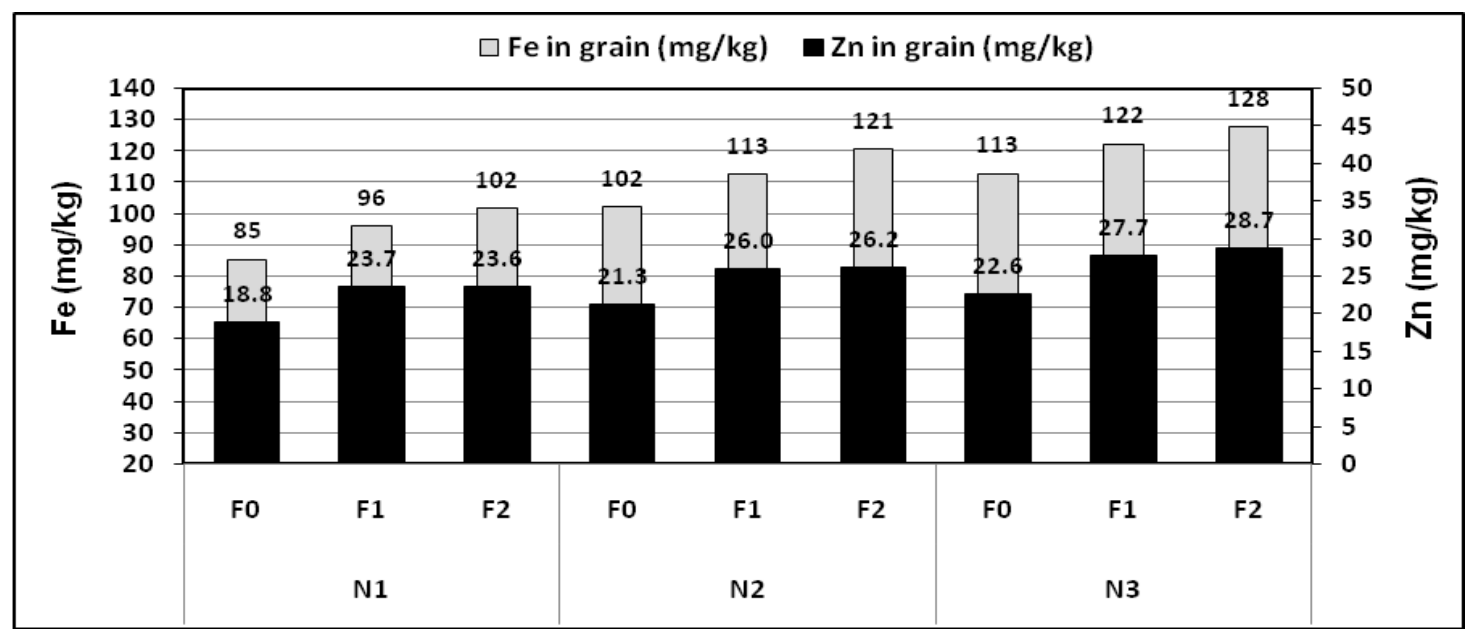

Fig. 3: Effect of interaction between $\mathrm{NR}$ and foliar micronutrients on $\mathrm{Fe}$ and $\mathrm{Zn}$ concentrations in grain.

\section{NPK-uptake $\left(\mathrm{kg} \mathrm{fed}^{-1}\right)$ :}

Data in Table 5 show that application of $\mathrm{NR}\left(\mathrm{N}_{1}, \mathrm{~N}_{2}\right.$ and $\left.\mathrm{N}_{3}\right)$ significantly increased $\mathrm{N}$, $\mathrm{P}$ and $\mathrm{K}$-uptake in wheat straw and grain yields in both seasons. The highest values of $\mathrm{N}, \mathrm{P}$ and $\mathrm{K}$ - uptake in straw and grain yield were obtained by application of $\mathrm{NR}-\mathrm{N}_{3}$ $\left(75 \mathrm{~kg} \mathrm{~N} \mathrm{fed}^{-1}\right)$. These values of N, P and Kuptake are related with the previous results of growth and yield, which were the highest at NR-N3. These results agreed with Nassar et al., (2004).

Also, data show that foliar application of chelated micronutrients Fe-EDTA, Zn-EDTA and Mn-EDTA significantly increased the uptake of $\mathrm{N}, \mathrm{P}$ and $\mathrm{K}$ in both seasons, by increasing number of sprays, but $\mathrm{P}$ and $\mathrm{K}$ uptake by straw were not significantly affected. Generally three foliar sprays $\left(F_{2}\right)$ had significant effects on the uptake of N, P and $K$ for straw and grain, and the highest values of $\mathrm{N}, \mathrm{P}$ and $\mathrm{K}$ uptake were obtained by three foliar sprays of micronutrient. These results showed the importance of foliar spraying of micronutrients at heading stage of wheat which induced further increases in $\mathrm{N}, \mathrm{P}$ and $\mathrm{K}$ uptake by plant. These results are in accordance with El-Fouly et al., (2001), Mahrous et al., (2010) and Zeidan et al., (2010).

Concerning the effect of interaction, data obtained in the same Table (5) reveal that interaction between NR and foliar treatments of micronutrients significantly affected the uptake of $\mathrm{N}$ but not $\mathrm{P}$ and $\mathrm{K}$ uptake. The highest total $\mathrm{N}, \mathrm{P}$ and $\mathrm{K}$ uptake were recorded under the interaction of $\mathrm{N}_{3} \times \mathrm{F}_{2}$, and this effect agrees with its effect on wheat yield and its studied components. It is obvious from the results that foliar spray of chelated micronutrients Fe-EDTA, Zn-EDTA and Mn-EDTA at tillering, elongation and heading stages with NR fertilization at $75 \mathrm{~kg}$ $\mathrm{N} \mathrm{fed}{ }^{-1}\left(\mathrm{~N}_{3}\right)$ have integration effect on wheat plants and its productivity, and these may be return to the balance effect between macro and micronutrients and its requirements in plants (Hu and Schmidhalter, 2001). These results are in accordance with El-Fouly et al., (2001), Nassar et al., (2004) and Abu ElFotoh et al., (2006).

\section{Micronutrients Uptake:}

Data in Table 6 show that application of NR significantly affected the uptake of $\mathrm{Fe}$, $\mathrm{Zn}$ and $\mathrm{Mn}$ of wheat grain and straw. The highest uptake of $\mathrm{Fe}, \mathrm{Zn}$ and $\mathrm{Mn}$ were recorded with NR- $\mathrm{N}_{3}$, but these values of $\mathrm{Fe}$, $\mathrm{Zn}$ and $\mathrm{Mn}$-content were decreased with $\mathrm{N}$ rates $N_{2}$ and $N_{1}$. These results may be attributed to synergism correlation between $\mathrm{N}$ and these elements. The results are in agreement with those obtained by Nassar et al., (2004). 
Also, data in same Table reveal that the uptake of $\mathrm{Fe}, \mathrm{Zn}$ and $\mathrm{Mn}$ of wheat grain and straw were significantly increased with foliar spray of micronutrients, but these increases were the highest with three foliar sprays $\left(F_{2}\right)$, where the differences between $F_{2}$ and $F_{1}$ were significant for $\mathrm{Fe}, \mathrm{Zn}$ and Mn-uptake. It is obvious from this result that foliar sprays of micronutrients at heading stage had significant effects on plant growth and its micronutrients contents, and showing the importance of foliar micronutrients at heading stage. This was in accordance with the results obtained by Habib (2009) and Ezatollah et al., (2016) who showed the importance of foliar spray at heading stage.
As for the effect of interaction, data in Table (6) illustrate that values of $\mathrm{Fe}, \mathrm{Zn}$ and $\mathrm{Mn}$ uptake in wheat straw yield, significantly increased with interaction between NR and foliar spray of micronutrients, but Fe-uptake in grain in both seasons, $\mathrm{Zn}$-uptake in grain in $2^{\text {nd }}$ seasons and Mn-uptake in grain in $1^{\text {st }}$ season were insignificant. The highest values of $\mathrm{Fe}, \mathrm{Zn}$ and $\mathrm{Mn}$ uptake in wheat grain and straw were with interaction of $\mathrm{N}_{3} \times$ $F_{2}$, and these results show the integration and the balance of fertilizer supplies from $\mathrm{N}$ fertilizer and micronutrients foliar spray on plant. These results agreed with Nassar et al., (2004), Abu El-Fotoh et al., (2006) and Ezatollah et al., (2016).

Table 5: Effect of nitrogen and micronutrients fertilization on the uptake of nitrogen, phosphorus and potassium by wheat plants grown in salted affected soils.

\begin{tabular}{|c|c|c|c|c|c|c|c|c|c|c|c|c|c|}
\hline \multirow{3}{*}{\multicolumn{2}{|c|}{$\begin{array}{l}\text { Characteristics } \\
\text { Treatments }\end{array}$}} & \multicolumn{6}{|c|}{$1^{\text {st }}$ season } & \multicolumn{6}{|c|}{$2^{\text {nd }}$ season } \\
\hline & & \multicolumn{2}{|c|}{$\begin{array}{l}\text { N-Uptake } \\
\left(\mathrm{kg} \mathrm{fed}^{-1}\right)\end{array}$} & \multicolumn{2}{|c|}{$\begin{array}{l}\text { P-Uptake } \\
\left(\mathrm{kg} \mathrm{fed}^{-1}\right)\end{array}$} & \multicolumn{2}{|c|}{$\begin{array}{l}\text { K-Uptake } \\
\left(\mathrm{kg} \mathrm{fed}^{-1}\right)\end{array}$} & \multicolumn{2}{|c|}{$\begin{array}{l}\text { N-Uptake } \\
\left(\mathrm{kg} \mathrm{fed}^{-1}\right)\end{array}$} & \multicolumn{2}{|c|}{$\begin{array}{l}\text { P-Uptake } \\
\left(\mathrm{kg} \mathrm{fed}^{-1}\right)\end{array}$} & \multicolumn{2}{|c|}{$\begin{array}{l}\text { K- Uptake } \\
\left(\mathrm{kg} \mathrm{fed}^{-1}\right)\end{array}$} \\
\hline & & Straw & Grain & Straw & Grain & Straw & Grain & Straw & Grain & Straw & Grain & Straw & Grain \\
\hline \multicolumn{14}{|c|}{ Nitrogen rates effect: } \\
\hline \multicolumn{2}{|c|}{$\mathrm{N}_{1}$} & $55.8^{c}$ & $48.2^{C}$ & $3.5^{\mathrm{c}}$ & $7.2^{c}$ & $33.9^{c}$ & $6.8^{c}$ & $53.0^{c}$ & $49.6^{c}$ & $4.2^{b}$ & $8.0^{c}$ & $40.6^{b}$ & $7.7^{\mathrm{C}}$ \\
\hline \multicolumn{2}{|c|}{$\mathrm{N}_{2}$} & $71.9^{\mathrm{b}}$ & $63.0^{\mathrm{b}}$ & $4.1^{b}$ & $9.8^{b}$ & $40.3^{b}$ & $9.1^{b}$ & $73.9^{b}$ & $65.6^{\mathrm{b}}$ & $4.5^{\mathrm{b}}$ & $10.8^{\mathrm{b}}$ & $47.3^{a}$ & $10.3^{b}$ \\
\hline \multicolumn{2}{|c|}{$\mathrm{N}_{3}$} & $87.6^{\mathrm{a}}$ & $74.3^{\mathrm{a}}$ & $4.9^{\mathrm{a}}$ & $11.1^{\mathrm{a}}$ & $45.2^{\mathrm{a}}$ & $10.7^{\mathrm{a}}$ & $92.8^{\mathrm{a}}$ & $76.5^{\mathrm{a}}$ & $5.8^{\mathrm{a}}$ & $12.1^{\mathrm{a}}$ & $49.7^{\mathrm{a}}$ & $12.1^{\mathrm{a}}$ \\
\hline \multicolumn{14}{|c|}{ Micronutrients effect: } \\
\hline \multicolumn{2}{|c|}{$\mathrm{F}_{0}$} & $63.2^{c}$ & $53.9^{c}$ & $4.0^{\mathrm{a}}$ & $8.4^{\mathrm{C}}$ & $36.4^{\mathrm{c}}$ & $7.8^{\mathrm{c}}$ & $61.3^{c}$ & $54.8^{c}$ & $4.0^{b}$ & $9.2^{c}$ & $38.6^{b}$ & $8.4^{\mathrm{C}}$ \\
\hline \multicolumn{2}{|c|}{$F_{1}$} & $72.9^{b}$ & $63.8^{b}$ & $4.2^{\mathrm{a}}$ & $9.5^{\mathrm{b}}$ & $38.9^{b}$ & $9.1^{b}$ & $77.4^{b}$ & $65.8^{b}$ & $5.3^{a}$ & $10.5^{b}$ & $49.5^{\mathrm{a}}$ & $10.4^{b}$ \\
\hline \multicolumn{2}{|c|}{$\mathrm{F}_{2}$} & $79.2^{\mathrm{a}}$ & $67.8^{\mathrm{a}}$ & $4.3^{a}$ & $10.2^{\mathrm{a}}$ & $44.0^{\mathrm{a}}$ & $9.8^{a}$ & $81.0^{a}$ & $71.1^{a}$ & $5.2^{\mathrm{a}}$ & $11.1^{\mathrm{a}}$ & $49.6^{a}$ & $11.2^{\mathrm{a}}$ \\
\hline \multicolumn{14}{|c|}{ Interaction effect: } \\
\hline \multirow{3}{*}{$\mathrm{N}_{1}$} & $\mathrm{~F}_{0}$ & $45.1^{g}$ & 41.3 & 3.3 & 6.1 & $29.2^{g}$ & 5.8 & $45.2^{e}$ & $41.5^{g}$ & 3.6 & $6.8^{f}$ & 35.1 & 6.4 \\
\hline & $F_{1}$ & $58.9^{f}$ & 49.6 & 3.8 & 7.3 & $32.5^{\dagger}$ & 7.2 & $55.3^{d}$ & $51.6^{f}$ & 4.6 & $8.3^{\mathrm{e}}$ & 42.7 & 8.1 \\
\hline & $\mathrm{F}_{2}$ & $63.5^{\text {ef }}$ & 53.7 & 3.5 & 8.1 & $39.9^{\text {de }}$ & 7.5 & $58.5^{\mathrm{cd}}$ & $55.8^{\mathrm{e}}$ & 4.5 & $8.8^{\mathrm{e}}$ & 43.9 & 8.6 \\
\hline \multirow{3}{*}{$\mathrm{N}_{2}$} & $\mathrm{~F}_{0}$ & $66.6^{\mathrm{e}}$ & 54.8 & 3.9 & 8.8 & $38.4^{\mathrm{e}}$ & 8.0 & $62.8^{c}$ & $54.0^{\text {ef }}$ & 3.9 & $9.7^{d}$ & 38.4 & 8.4 \\
\hline & $\mathrm{F}_{1}$ & $72.4^{d}$ & 65.5 & 4.0 & 10.0 & $40.4^{d}$ & 9.1 & $77.2^{b}$ & $67.9^{d}$ & 4.7 & $10.9^{b c}$ & 51.1 & 10.8 \\
\hline & $\mathrm{F}_{2}$ & $76.7^{\mathrm{cd}}$ & 68.9 & 4.3 & 10.6 & $42.0^{b c}$ & 10.2 & $81.7^{b}$ & $74.7^{\mathrm{c}}$ & 5.1 & $11.7^{\mathrm{a}}$ & 52.5 & 11.6 \\
\hline \multirow{3}{*}{$\mathrm{N}_{3}$} & $\mathrm{~F}_{0}$ & $78.1^{c}$ & 65.6 & 4.9 & 10.3 & $41.7^{\mathrm{cd}}$ & 9.5 & $76.0^{b}$ & $69.0^{d}$ & 4.7 & $11.2^{c}$ & 42.3 & 10.4 \\
\hline & $F_{1}$ & $87.5^{\mathrm{b}}$ & 76.4 & 4.9 & 11.2 & $43.7^{b}$ & 10.9 & $99.6^{a}$ & $77.8^{b}$ & 6.6 & $12.3^{b}$ & 54.6 & 12.4 \\
\hline & $F_{2}$ & $97.4^{\mathrm{a}}$ & 80.9 & 5.0 & 11.8 & $50.0^{a}$ & 11.7 & $102.9^{a}$ & $82.8^{a}$ & 6.2 & $12.9^{\mathrm{a}}$ & 52.3 & 13.4 \\
\hline \multicolumn{14}{|c|}{ LSD at $5 \%$ : } \\
\hline \multicolumn{2}{|c|}{ Nitrogen } & 2.15 & 2.42 & 0.23 & 0.21 & 0.91 & 0.66 & 3.50 & 2.03 & 0.41 & 0.40 & 3.93 & 0.48 \\
\hline \multirow{2}{*}{\multicolumn{2}{|c|}{$\begin{array}{l}\text { Micronutrients } \\
\text { Interaction }\end{array}$}} & 2.85 & 1.91 & Ns & 0.18 & 1.07 & 0.33 & 3.64 & 1.68 & 0.33 & 0.14 & 2.56 & 0.43 \\
\hline & & 4.95 & Ns & Ns & Ns & 1.85 & Ns & 6.31 & 2.91 & Ns & Ns & Ns & Ns \\
\hline
\end{tabular}


Balanced fertilization of nitrogen and micronutrients for wheat grown...........

Table 6: Effect of nitrogen and micronutrients fertilization on the uptake of iron, manganese and zinc by wheat plants grown in salted affected soils.

\begin{tabular}{|c|c|c|c|c|c|c|c|c|c|c|c|c|c|}
\hline \multirow{3}{*}{\multicolumn{2}{|c|}{$\begin{array}{l}\text { Characteristics } \\
\text { Treatments }\end{array}$}} & \multicolumn{6}{|c|}{$1^{\text {st }}$ season } & \multicolumn{6}{|c|}{$2^{\text {nd }}$ season } \\
\hline & & \multicolumn{2}{|c|}{$\begin{array}{l}\text { Fe-Uptake } \\
\left(\mathrm{kg} \mathrm{fed}^{-1}\right)\end{array}$} & \multicolumn{2}{|c|}{$\begin{array}{l}\text { Mn-Uptake } \\
\left(\mathrm{kg} \mathrm{fed}^{-1}\right)\end{array}$} & \multicolumn{2}{|c|}{$\begin{array}{c}\text { Zn-Uptake } \\
\left(\mathrm{kg} \mathrm{fed}^{-1}\right)\end{array}$} & \multicolumn{2}{|c|}{$\begin{array}{l}\text { Fe-Uptake } \\
\left(\mathrm{kg} \mathrm{fed}^{-1}\right)\end{array}$} & \multicolumn{2}{|c|}{$\begin{array}{l}\text { Mn-Uptake } \\
\left(\mathrm{kg} \mathrm{fed}^{-1}\right)\end{array}$} & \multicolumn{2}{|c|}{$\begin{array}{c}\text { Zn-Uptake } \\
\left(\mathrm{kg} \mathrm{fed}^{-1}\right)\end{array}$} \\
\hline & & Straw & Grain & Straw & Grain & Straw & Grain & Straw & Grain & Straw & Grain & Straw & Grain \\
\hline \multicolumn{14}{|c|}{ Nitrogen Rates Effect: } \\
\hline & & $1.072^{c}$ & $0.179^{\mathrm{C}}$ & $0.196^{\mathrm{C}}$ & & & $0.042^{c}$ & $1.232^{\mathrm{C}}$ & $0.186^{\mathrm{C}}$ & & $0.062^{c}$ & $0.084^{c}$ & $0.044^{c}$ \\
\hline & & $1.364^{\mathrm{b}} \mathrm{C}$ & $0.284^{b}$ & $0.249^{b}$ & $0.076^{\mathrm{b}}$ & $0.146^{b}$ & $0.063^{b}$ & $1.888^{\mathrm{b}}$ & $0.262^{b}$ & 0.24 & $0.081^{b}$ & $0.138^{\mathrm{b}}$ & $0.057^{\mathrm{b}}$ \\
\hline & & $1.868^{\mathrm{a}}$ & 0.33 & 0.36 & & 0.18 & $0.078^{\mathrm{a}}$ & $2.214^{\mathrm{a}}$ & 0.3 & 0.33 & $0.106^{\mathrm{a}}$ & $0.196^{\mathrm{a}}$ & $0.067^{\mathrm{a}}$ \\
\hline \multicolumn{14}{|c|}{ Micronutrients Effect: } \\
\hline & & $1.182^{\mathrm{C}}$ & 0.22 & $0.194^{\mathrm{C}}$ & 0.06 & $0.105^{\mathrm{c}}$ & $0.051^{\mathrm{c}}$ & $1.501^{\mathrm{C}}$ & $0.214^{c}$ & $0.196^{c}$ & $0.070^{\mathrm{C}}$ & $0.104^{\mathrm{C}}$ & $0.041^{\mathrm{C}}$ \\
\hline & & $1.425^{\mathrm{b}} \mathrm{C}$ & $0.273^{b}$ & $0.266^{b}$ & 0.075 & $0.149^{b}$ & $0.063^{b}$ & $1.837^{\mathrm{b}}$ & 0.26 & 0.25 & $0.085^{b}$ & $0.146^{\mathrm{b}}$ & $0.062^{b}$ \\
\hline & & $1.698^{\mathrm{a}}$ & 0.29 & $0.350^{\mathrm{a}}$ & & $0.182^{\mathrm{a}}$ & $0.069^{\mathrm{a}}$ & $1.995^{\mathrm{a}}$ & 0.29 & 0.30 & $0.094^{\mathrm{a}}$ & $0.168^{\mathrm{a}}$ & $0.065^{\mathrm{a}}$ \\
\hline \multicolumn{14}{|c|}{ Interaction Effect: } \\
\hline \multirow{3}{*}{$\mathrm{N}_{1}$} & $\mathrm{~F}_{0}$ & $0.830^{f}$ & 0.147 & $0.132^{g}$ & 0.047 & $0.065^{9}$ & $0.035^{f}$ & $1.038^{\mathrm{h}}$ & 0.148 & 0.1 & $0.053^{\dagger}$ & $0.066^{9}$ & 0.030 \\
\hline & $F_{1}$ & $1.064^{\mathrm{e}}$ & 0.185 & $0.193^{f}$ & 0.057 & $0.112^{f}$ & $0.044^{e}$ & $1.257^{\mathrm{g}}$ & 0.192 & $0.170^{g}$ & $0.066^{\mathrm{e}}$ & $0.088^{f}$ & 0.049 \\
\hline & $\mathrm{F}_{2}$ & $1.322^{d}$ & 0.206 & $0.265^{d}$ & 0.063 & $0.141^{\mathrm{e}}$ & $0.047^{\mathrm{e}}$ & $1.401^{f}$ & 0.217 & $0.206^{\mathrm{e}}$ & $0.068^{\mathrm{e}}$ & $0.100^{\mathrm{e}}$ & 0.051 \\
\hline \multirow{3}{*}{$\mathrm{N}_{2}$} & $\mathrm{~F}_{0}$ & $1.225^{\mathrm{e}}$ & 0.236 & $0.191^{f}$ & 0.067 & $0.109^{f}$ & $0.053^{d}$ & $1.507^{\mathrm{e}}$ & 0.217 & $0.194^{f}$ & $0.067^{\mathrm{e}}$ & $0.098^{\mathrm{e}}$ & 0.041 \\
\hline & $F_{1}$ & $1.348^{d}$ & 0.293 & $0.249^{\mathrm{e}}$ & 0.077 & $0.155^{d}$ & $0.065^{c}$ & $1.996^{d}$ & 0.263 & $0.257^{d}$ & $0.084^{d}$ & $0.146^{d}$ & 0.063 \\
\hline & $\mathrm{F}_{2}$ & $1.520^{c}$ & 0.324 & $0.308^{c}$ & 0.082 & $0.174^{\mathrm{C}}$ & $0.069^{c}$ & $2.161^{c}$ & 0.306 & $0.295^{\mathrm{C}}$ & $0.093^{c}$ & $0.172^{c}$ & 0.068 \\
\hline \multirow{3}{*}{$\mathrm{N}_{3}$} & $\mathrm{~F}_{0}$ & $1.489^{c}$ & 0.304 & $0.260^{\text {de }}$ & 0.080 & $0.140^{\mathrm{e}}$ & $0.065^{c}$ & $1.959^{d}$ & 0.276 & $0.262^{d}$ & $0.091^{c}$ & $0.150^{d}$ & 0.051 \\
\hline & $F_{1}$ & $1.863^{\mathrm{b}}$ & 0.339 & $0.356^{b}$ & 0.091 & $0.181^{b}$ & $0.079^{b}$ & $2.259^{b}$ & 0.334 & $0.349^{b}$ & $0.106^{b}$ & $0.205^{b}$ & 0.073 \\
\hline & $\mathrm{F}_{2}$ & $2.253^{\mathrm{a}}$ & 0.364 & $0.479^{\mathrm{a}}$ & 0.103 & $0.232^{\mathrm{a}}$ & $0.089^{a}$ & $2.423^{a}$ & 0.374 & $0.405^{a}$ & $0.122^{\mathrm{a}}$ & $0.233^{\mathrm{a}}$ & 0.076 \\
\hline \multicolumn{14}{|c|}{ SD at $5 \%:$} \\
\hline \multicolumn{2}{|c|}{ Nitrogen } & 0.035 & 0.017 & 0.021 & 0.002 & 0.012 & 0.002 & 0.018 & 0.025 & 0.005 & 0.007 & 0.003 & 0.004 \\
\hline \multirow{2}{*}{\multicolumn{2}{|c|}{ Micronutrients }} & 0.043 & 0.018 & 0.007 & 0.002 & 0.005 & 0.003 & 0.038 & 0.019 & 0.007 & 0.004 & 0.006 & 0.002 \\
\hline & & 0.074 & Ns & 0.012 & Ns & 0.008 & 0.005 & 0.065 & Ns & 0.011 & 0.006 & 0.011 & Ns \\
\hline
\end{tabular}

\section{$\mathrm{K}$ and $\mathrm{Na} \%$ and $\mathrm{K} / \mathrm{Na}$ ratio:}

Data in Table 7 show that applications of NR at 50,75 and $100 \%$ of recommended dose $\left(75 \mathrm{~kg} \mathrm{~N} \mathrm{fed}^{-1}\right)$ had significant effects on $\mathrm{K}$ and $\mathrm{Na} \%$ in straw and grain, but this effect was insignificant for $\mathrm{K} \%$ in grain in $1^{\text {st }}$ season. $\mathrm{K}$ concentration slightly decreased in straw with increasing $\mathrm{N}$ rate, but the total content of straw were significantly increased as shown in Table $7 . \mathrm{K} \%$ in grain significantly increased with increasing $\mathrm{N}$ application rate up to $\mathrm{N}_{3}$, while, $\mathrm{Na}$ concentrations in straw and grain were decreased significantly with increasing NR. As for $\mathrm{K} / \mathrm{Na}$ ratio, it is clear from Table 7 that the highest ratio of $\mathrm{K} / \mathrm{Na}$ was $6.31,6.36$ in the $1^{\text {st }}$ season and 7.05 and 4.71 in the $2^{\text {nd }}$ season for straw and grain, respectively with $\mathrm{N}_{3}$. In this regard, the previous researches showed that increasing $\mathrm{K} / \mathrm{Na}$ ratio in plant is considered an indicator for improving plant growth and enhancing plant tolerant to salinity (El-Fouly et al., 2001, Ghogdi et al., 2012 and El-Dissoky 2013). 
El-Dissoky, et al.,

Table 7: Effect of nitrogen and micro nutrients $\mathrm{Fe}, \mathrm{Mn}$ and $\mathrm{Zn}$ fertilization on concentrations of $\mathrm{K}$ and $\mathrm{Na}$ and $\mathrm{K} / \mathrm{Na}$ ratio for wheat straw and grain grown in salted affected soils.

\begin{tabular}{|c|c|c|c|c|c|c|c|c|c|c|c|c|c|}
\hline \multirow{3}{*}{\multicolumn{2}{|c|}{$\begin{array}{l}\text { Characteristics } \\
\text { Treatments }\end{array}$}} & \multicolumn{6}{|c|}{$1^{\text {st }}$ season } & \multicolumn{6}{|c|}{$2^{\text {nd }}$ season } \\
\hline & & \multicolumn{2}{|c|}{$K \%$} & \multicolumn{2}{|c|}{$\mathrm{Na} \%$} & \multicolumn{2}{|c|}{$\begin{array}{l}\mathrm{K} / \mathrm{Na} \\
\text { Ratio }\end{array}$} & \multicolumn{2}{|c|}{$\mathrm{K} \%$} & \multicolumn{2}{|c|}{$\mathrm{Na} \%$} & \multicolumn{2}{|c|}{$\begin{array}{l}\mathrm{K} / \mathrm{Na} \\
\text { Ratio }\end{array}$} \\
\hline & & Straw & Grain & Straw & Grain & Straw & Grain & Straw & Grain & Straw & Grain & Straw & Grain \\
\hline \multicolumn{14}{|c|}{ litrogen rates effect: } \\
\hline \multicolumn{2}{|c|}{$\mathrm{N}_{1}$} & $1.02^{\mathrm{a}}$ & 0.37 & $0.189^{a}$ & $0.112^{\mathrm{a}}$ & $5.47^{\mathrm{b}}$ & $3.62^{\mathrm{c}}$ & $1.10^{\mathrm{a}}$ & $0.38^{\mathrm{c}}$ & $0.163^{\mathrm{a}}$ & $0.102^{a}$ & 6.78 & $3.43^{\mathrm{C}}$ \\
\hline \multicolumn{2}{|c|}{$\mathrm{N}_{2}$} & $1.01^{\mathrm{a}}$ & 0.39 & $0.171^{b}$ & $0.106^{\mathrm{a}}$ & $5.95^{\mathrm{ab}}$ & $5.00^{b}$ & $0.99^{b}$ & $0.40^{b}$ & $0.149^{b}$ & $0.078^{b}$ & 6.67 & $3.84^{\mathrm{b}}$ \\
\hline \multicolumn{2}{|c|}{$\mathrm{N}_{3}$} & $0.95^{\mathrm{b}}$ & 0.40 & $0.151^{\mathrm{c}}$ & $0.091^{b}$ & $6.31^{a}$ & 6.36 & $0.95^{\mathrm{b}}$ & $0.43^{\mathrm{a}}$ & $0.135^{\circ}$ & $0.064^{\mathrm{c}}$ & $7.05^{\mathrm{a}}$ & $4.71^{\mathrm{a}}$ \\
\hline \multicolumn{14}{|c|}{ Micronutrients effect: } \\
\hline \multicolumn{2}{|c|}{$\mathrm{F}_{0}$} & $1.01^{\mathrm{a}}$ & $0.37^{\circ}$ & $0.188^{a}$ & 0.106 & $5.41^{\mathrm{C}}$ & $4.45^{\mathrm{C}}$ & $0.95^{\mathrm{b}}$ & $0.37^{b}$ & $0.157^{\mathrm{a}}$ & 0.086 & $6.04^{\mathrm{b}}$ & $3.53^{\mathrm{C}}$ \\
\hline \multicolumn{2}{|c|}{$F_{1}$} & $0.97^{\mathrm{b}}$ & $0.39^{\mathrm{b}}$ & $0.167^{b}$ & 0.104 & $5.85^{\mathrm{b}}$ & $5.10^{b}$ & $1.06^{\mathrm{a}}$ & $0.42^{\mathrm{a}}$ & $0.149^{b}$ & 0.081 & $7.20^{\mathrm{a}}$ & $4.06^{\mathrm{b}}$ \\
\hline \multicolumn{2}{|c|}{$\mathrm{F}_{2}$} & $1.01^{\mathrm{a}}$ & $0.40^{\mathrm{a}}$ & $0.156^{\mathrm{C}}$ & 0.098 & $6.48^{\mathrm{a}}$ & $5.43^{\mathrm{a}}$ & $1.02^{\mathrm{a}}$ & $0.42^{\mathrm{a}}$ & $0.141^{\mathrm{C}}$ & 0.079 & $7.26^{\mathrm{a}}$ & $4.38^{\mathrm{a}}$ \\
\hline \multicolumn{14}{|c|}{ Interaction effect: } \\
\hline \multirow{3}{*}{$\mathrm{N}_{1}$} & $\mathrm{~F}_{0}$ & $1.03^{b}$ & 0.35 & $0.213^{a}$ & 0.115 & $4.83^{\mathrm{e}}$ & 3.38 & 1.05 & 0.35 & 0.174 & 0.104 & 6.11 & 3.08 \\
\hline & $F_{1}$ & $0.96^{\mathrm{c}}$ & 0.38 & $0.183^{c}$ & 0.113 & $5.23^{d}$ & 3.74 & 1.13 & 0.39 & 0.165 & 0.102 & 6.88 & 3.49 \\
\hline & $\mathrm{F}_{2}$ & $1.08^{\mathrm{a}}$ & 0.38 & $0.171^{d}$ & 0.107 & $6.36^{b}$ & 3.73 & 1.10 & 0.40 & 0.150 & 0.101 & 7.35 & 3.71 \\
\hline \multirow{3}{*}{$\mathrm{N}_{2}$} & $\mathrm{~F}_{0}$ & $1.03^{b}$ & 0.37 & $0.192^{b}$ & 0.107 & $5.35^{d}$ & 4.51 & 0.91 & 0.36 & 0.156 & 0.083 & 5.80 & 3.38 \\
\hline & $F_{1}$ & $1.02^{\mathrm{b}}$ & 0.38 & $0.166^{\mathrm{e}}$ & 0.109 & $6.13^{b c}$ & 5.03 & 1.05 & 0.42 & 0.147 & 0.077 & 7.15 & 3.90 \\
\hline & $\mathrm{F}_{2}$ & $0.99^{b c}$ & 0.41 & $0.156^{\mathrm{fg}}$ & 0.101 & $6.37^{\mathrm{b}}$ & 5.44 & 1.01 & 0.43 & 0.143 & 0.075 & 7.06 & 4.23 \\
\hline \multirow{3}{*}{$\mathrm{N}_{3}$} & $\mathrm{~F}_{0}$ & $0.96^{\mathrm{c}}$ & 0.38 & $0.160^{\dagger}$ & 0.095 & $6.03^{c}$ & 5.46 & 0.88 & 0.39 & 0.141 & 0.070 & 6.22 & 4.13 \\
\hline & $F_{1}$ & $0.93^{d}$ & 0.41 & $0.151^{\mathrm{g}}$ & 0.091 & $6.19^{b c}$ & 6.52 & 1.01 & 0.44 & 0.134 & 0.063 & 7.56 & 4.79 \\
\hline & $\mathrm{F}_{2}$ & $0.95^{\mathrm{c}}$ & 0.42 & $0.142^{\mathrm{h}}$ & 0.087 & $6.71^{\mathrm{a}}$ & 7.10 & 0.96 & 0.45 & 0.130 & 0.059 & 7.38 & 5.19 \\
\hline \multicolumn{14}{|c|}{ LSD at $5 \%$} \\
\hline \multirow{3}{*}{\multicolumn{2}{|c|}{$\begin{array}{l}\text { Nitrogen } \\
\text { Micronutrients } \\
\text { Interaction }\end{array}$}} & 0.06 & Ns & 0.011 & 0.008 & 0.59 & 0.92 & 0.07 & 0.01 & 0.009 & 0.009 & Ns & 0.27 \\
\hline & & 0.03 & 0.01 & 0.003 & 0.005 & 0.17 & 0.42 & 0.06 & 0.02 & 0.006 & Ns & 0.48 & 0.25 \\
\hline & & 0.05 & Ns & 0.006 & Ns & 0.29 & Ns & Ns & Ns & Ns & Ns & Ns & Ns \\
\hline
\end{tabular}

Foliar sprays of chelated micronutrients had a positive effect on $\mathrm{K}$ and $\mathrm{Na}$ concentrations, where $\mathrm{K} \%$ was increased but $\mathrm{Na} \%$ was decreased with foliar spray of Fe-EDTA, Zn-EDTA and Mn-EDTA treatments which consequently reflected on $\mathrm{K} / \mathrm{Na}$ ratio increments in straw and grain. As for $\mathrm{K} / \mathrm{Na}$ ratio the superiority was for the three foliar sprays of micronutrients $\left(F_{2}\right)$ compared with control $\left(F_{0}\right)$. Interaction between NR and foliar micronutrients treatments significantly affected $\mathrm{K}$ and $\mathrm{Na} \%$ in straw in $1^{\text {st }}$ season, but it had insignificant effect in the $2^{\text {nd }}$ season (Table 7 and Figs 4 and 5). However, $\mathrm{K} \%$ in grain was increased and $\mathrm{Na} \%$ decreased with interaction that reflected on increases $\mathrm{K} / \mathrm{Na}$ ratio. The highest ratio of $\mathrm{K} / \mathrm{Na}$ in straw and grain was 6.71 and 7.10 in the $1^{\text {st }}$ season and 7.38 and 5.15 in the $2^{\text {nd }}$ season, respectively with interaction $\mathrm{N}_{3} \times \mathrm{F}_{2}$. These results agree with Ghogdi et al., (2012) and El-Dissoky (2013) who found that soil salinity significantly affected $\mathrm{K} / \mathrm{Na}$ ratio in straw and grain, where the values of $\mathrm{K} / \mathrm{Na}$ ratio decreased under saline soil compared with non saline soil. 
It can be concluded that application of $100 \%$ RN $\left(75 \mathrm{~kg} \mathrm{~N} \mathrm{Fed}^{-1}\right)$ along with three foliar sprays of chelated micronutrients $\mathrm{Fe}$ EDTA, Zn-EDTA and Mn-EDTA was the optimum for wheat growth, straw and grain yields, where the interaction effect between them was the highest for wheat yield characteristics, grain and straw yields, grain quality, the uptake of $\mathrm{N}, \mathrm{P}, \mathrm{K}, \mathrm{Fe}, \mathrm{Zn}$ and $\mathrm{Mn}$ and $\mathrm{K} / \mathrm{Na}$ ratio. The results illustrate the importance of balanced fertilization of $\mathrm{N}$ and foliar micronutrients for plants grown in salted affected soil.

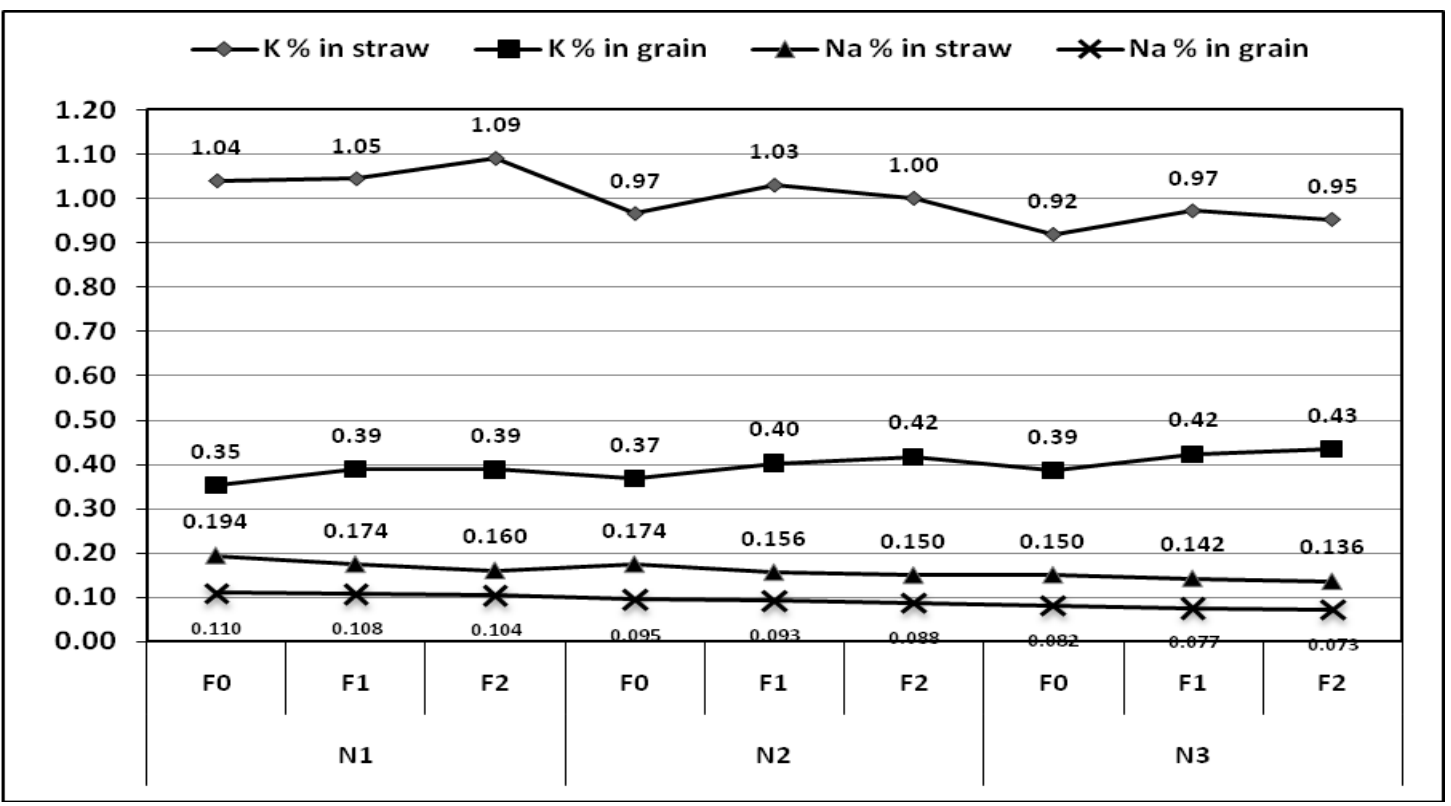

Fig. 4: Effect of interaction between NR and foliar micronutrients on Fe and Zn concentrations in grain.

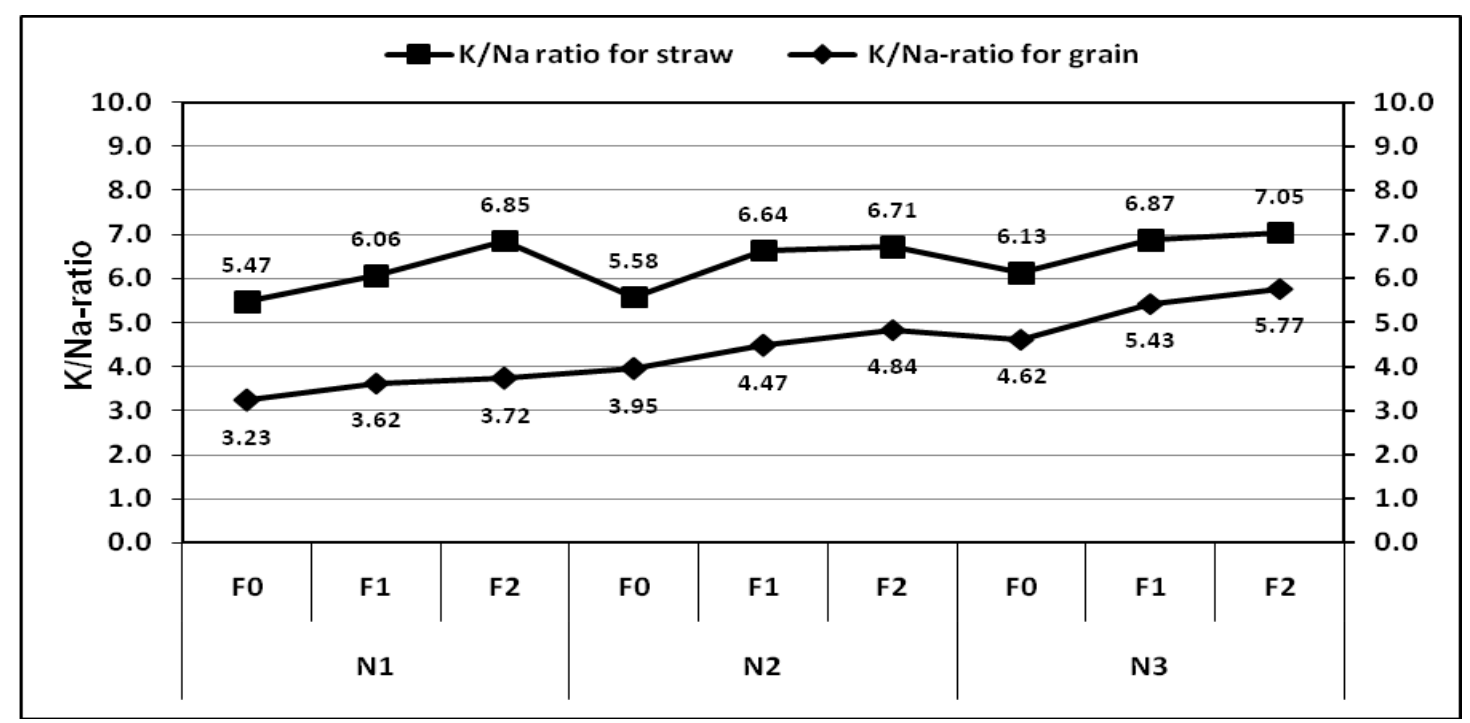

Fig. 5: Effect of interaction between $\mathrm{NR}$ and foliar micronutrients on $\mathrm{Fe}$ and $\mathrm{Zn}$ concentrations in grain. 


\section{Conclusion}

This study can be recommended by wheat crop fertilization under salt affected soils with $\mathrm{N}$-fertilization at $75 \mathrm{~kg} \mathrm{~N} \mathrm{Fed}^{-1}$ (225 kg ammonium nitrate fed $^{-1}$ nearly) with three foliar sprays of chelated micronutrients Fe-EDTA, Zn-EDTA and Mn-EDTA (0.3 $\mathrm{g} \mathrm{L}^{-1}$ of each chelate) at plant growth stages of tillering, elongation and heading with addition the constant recommended doses of phosphorus and potassium to achieve the highest grain yield with high quality under the same conditions.

\section{REFERENCES}

Abd El-Hadi, A. H. (2015). Effect of Zn, Mn and Fe-chelates and some different foliar fertilizers on the production of wheat, potato and sugarcane under Egyptian conditions. Advances in Environ. Biology J., 9(24):229-233.

Abd El-Magid, A. A. (2001). Response of barly to foliar application of some micronutrients under different level of soil salinity. J. Agric. Mansoura Univ., 26(11):7411-7422.

Abu El-Fotoh, H. G., Manal A. Ataya, A. A. Abd El-Magid, M. A. El-Akabawy and Nadia O. Monged (2006). The effectiveness of foliar spraying with putrescine and micronutrients for improving the yield of wheat and rice crops under soil salinity conditions. Egypt J. of Appl. Sci., 21(12A):321-328.

Bakry, A.B., M. H. Taha, M. F. El-Karamany and M. T. Said (2016). Improving productivity and quality of two wheat cultivars using humic acid and zinc foliar application under sandy soil conditions. Res. J. of Pharmaceutical, Biological and Chemical Sci., 7(3):606-616.

El-Dissoky, R. A. (2013). Effect of nitrogen, phosphorus and potassium application as soil and foliar on wheat productivity at soil salinity conditions. J. Soil Sci. and Agric. Eng., Mansoura Univ., 4 (8): 647 660.

El-Fouly, M. M., M. M. Zeinab and A. S. Zeinab (2001). Micronutrients spray as a tool to increase tolerance of faba been and wheat plants to salinity. Plant Nutrition J. 92(6):422-423.

Ezatollah, E., M. Abdoli, S. B. Mousavi and B. Sadeghzadeh (2016). Impact of foliar zinc application on agronomic traits and grain quality parameters of wheat grown in zinc deficient soil. Ind. J. Plant Physiol. Published online 30 May 2016.

Faizy, S. E-D. A., M. M. A. Amer and M. M. Ragap (2010). Response of wheat yield and apparent nitrogen recovery of fertilizer to mineral nitrogen and biofertilizer application in salt affected soils. J. Agric. Res. Kafer El-Sheikh Univ., 36 (1):74-96.

FAO (2002). State of Food Insecurity in the World.

Ghogdi, E. A., A. I. Darbandi and A. Borzouei (2012). Effects of salinity on some physiological traits in wheat (Triticum aestivum L.) cultivars. Indian J. Sci. and Technol., 5(1):1901-1906.

Gomez, K.A. and A.A. Gomez (1984). Statistical Procedures for Agriculture Research. 2nd Ed., John Wiley and Sons.

Habib, M. (2009). Effect of foliar application of $\mathrm{Zn}$ and $\mathrm{Fe}$ on wheat yield and quality. African J. of Biotechnology, 8(24):67956798.

$\mathrm{Hu}, \mathrm{Y}$. and U. Schmidhalter (2001). Effects of salinity and macronutrient levels on micronutrients in wheat. $J$. of Plant Nutrition, 24(2):273-281.

Jackson, M. L. (1967). Soil Chemical Analysis. Prentic Hall of land on Private Lim. Indian private Limited, New Delhi.

Ling, F. and M. Silberbush (2002). Response of maize to foliar versus soil application of nitrogen-phosphoruspotassium fertilizers. J. of Plant Nutrition 11:2333-2342.

Mahrous, N. M., Amany M. Sallam, Jaclin G. Sadek and K. A. Shaban (2010). Improving wheat production and grain quality by inorganic, organic and some micronutrients fertilizers under saline 
condition. Research J. OF Agric. And Biological Sci., 6(6): 1087-1098.

Marschner, H. (1995). "Mineral Nutrition of Higher Plants". $2^{\text {nd }} E d$. Academic Press.

Mengel, K. and E. A. Kirkby (2001). "Principles of Plant Nutrition", International Potash Institute, Bern, Switzerland.

Mosaad, I. S. M., E. E. E. Khafagy and R. A. El-Dissoky (2013). Effect of mineral, bio and organic nitrogen fertilization on wheat yield and nitrogen utilization efficiency and uptake at northern delta of Egypt. J. Soil Sci. and Agric. Eng., Mansoura Univ., 4(10): 1101 - 1116.

Nassar, K. E. M., M. M. El-Shouny and E. M. K. Behiry (2004). Improving the quantity and quality of wheat in salt affected soils. Zagazig J. Agric. Res., 31(6):2861-2883.

Negm, A. Y. and F. A. Zahran (2001). Optimizing time of micronutrient application to wheat plants grown on sandy soils. Egypt. J. Agric. Res., 79(3):813-823.

Page, A. L. (ED) (1982). Methods of Soil Analysis. Part2: Chemical and microbiological properties, (2nd Ed). Am. Soc. At Agron. Inc. Soil Sci. Soc. Of Am. Inc., Madison, Wisconsin, VSA.

Sairam, R. K. and A. Tyagi (2004). Physiology and molecular biology of salinity stress tolerance in plants. Current Sci. 86(3): 407-421.

Sharpley, A. N., J. J. Meisinger, J. F. Power and D. L. Suarez (1992). Root extraction of nutrients associated with long-term soil management. In advances in soil Sci. Steward, B., Eid; Springer: New York; 151-217.

Stepien, A. and K. Wajtkowiak (2016). Effect of foliar application of $\mathrm{Cu}, \mathrm{Zn}$ and $\mathrm{Mn}$ on yield and quality indicators of winter wheat grain. Chilean J. of Agric. Res. 76(2): April-June.

WHO (2002). The Report of World Health Organization; Geneva.

Zeidan, M. S., Manal F. Mohamed and H. A. Hamouda (2010). Effect of foliar fertilization of $\mathrm{Fe}, \mathrm{Mn}$ and $\mathrm{Zn}$ on wheat yield and quality in low sandy soils fertility. World J. Agic. Sci., 6(6): 696-699. 


\section{التسميد المتوازن من النتروجين والعناصر الصغرى للقمح المنزرع فى الأراضي المتأثرة بالأملاح}

رمضان عوض الاسوقي (1) ، رمضان عبد السلام رمضان(2) ، إبراهيم عبد المنعم حجاب(1) (1) معهد بحوث الأراضي والمياه والبيئة - مركز البحوث الزراعية - الجيزة - مصر (2) قسم بحوث القمح - معهد بحوث المحاصيل الحقلية - مركز البحوث الزراعية - الجيزة- مصر الملخص العربى

التسميد آلمنوازن من أهم الضروريات للزراعة المستدامة والإنتاج النباتي بالأراضي القديمة والحديثة، علاوة على ذللك فتوازن الإمداد بالأسمدة في حالة الزراعة تحت ظروف الإجهاد المختلفة وبالأخص الملحي أصبح من الأهمية

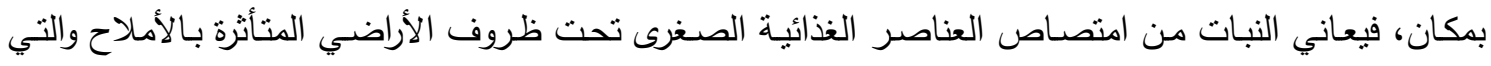

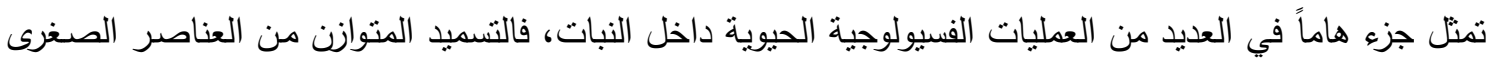
والنتروجين يعمل على تحسين وزيادة نمو النباتات وإنتاجية وجودة المحصول تحت مثل هذه الظروف، وعليه فقد

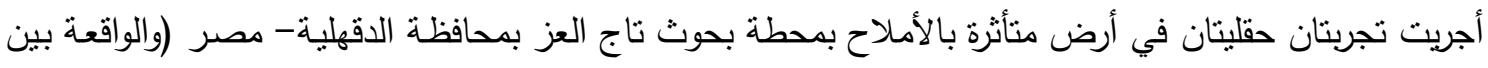

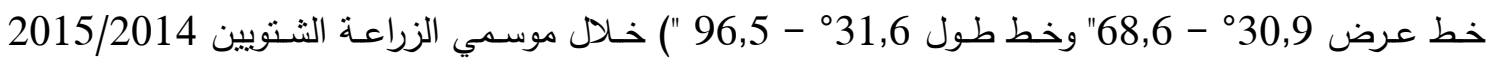
و2016/2015 لنقيم إضـافة مستويات مختلفة من النتروجين (50، 75 و 100 \% من النتروجين الموصي بـهـ

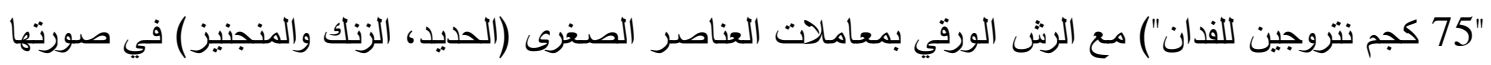
المخلبية (بدون رش، رش مرتين عند مرحلتي التفريع والاستطالة ورش ثناث مرات عند مراحل التفريع والاسنطالة

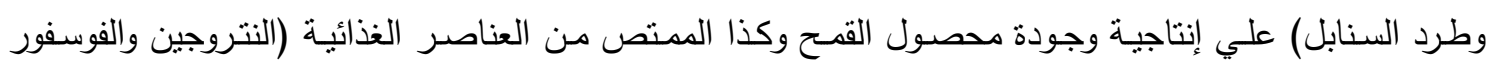

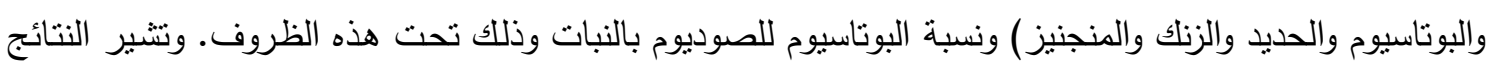

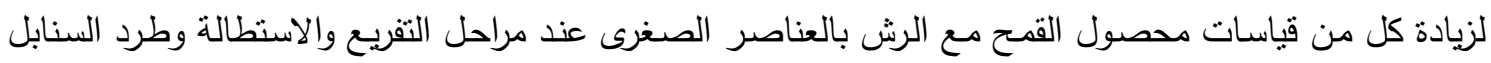
والتسميد الأزوتي عند معدل 100 \% من الموصي بـه (75 كجم نتروجين/ للفدان)، كذللك أنشارت النتائج لزيادة

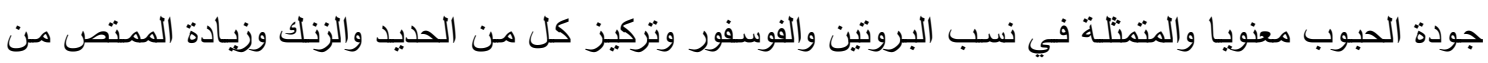

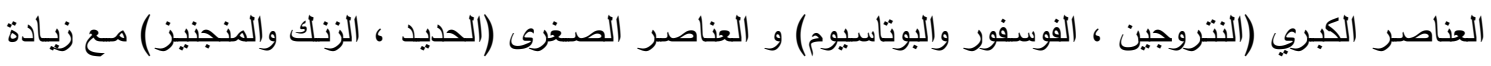

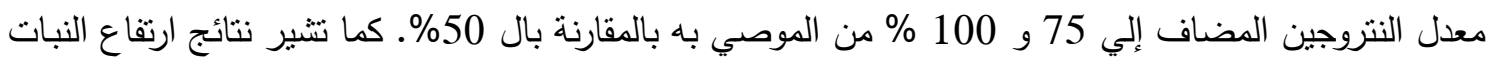
ومحصول الحبوب والقش وتركيز كل من البوتاسيوم والصسوديوم ونسب البوتاسيوم للصوديوم لتحسن نمو النبات

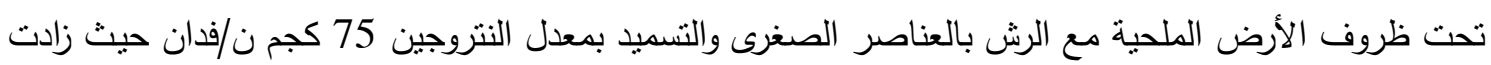

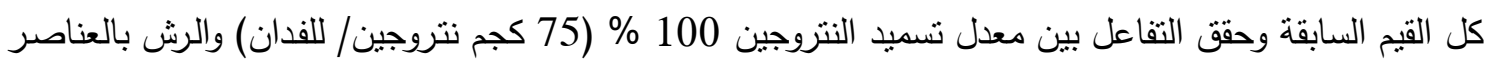

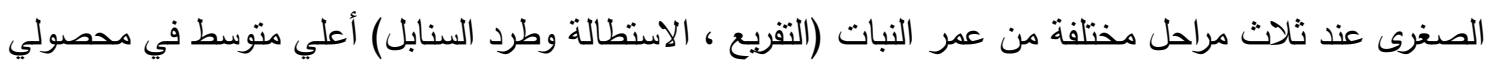
حبوب القمح ( 19,24 إردب/ للفدان) والقش (5368 كجم /للفدان) لكلا موسمي الزراعة. 